\title{
Chromatic Gain Controls in Visual Cortical Neurons
}

\author{
Samuel G. Solomon and Peter Lennie \\ Center for Neural Science, New York University, New York, New York 10003
}

\begin{abstract}
Although the response of a neuron in the visual cortex generally grows nonlinearly with contrast, the spatial tuning of the cell remains stable. This is thought to reflect the activity of a contrast gain control ("normalization") that has very broad tuning on the relevant stimulus dimension. Contrast invariant tuning on a particular dimension is probably necessary for reliable representation of stimuli on that dimension. In the lateral geniculate nucleus (LGN), V1, and V2 of anesthetized macaque, we measured chromatic tuning of neurons at several contrasts to characterize the gain controls and identify cells that might be important for representing color. We estimated separately the chromatic signature of the linear receptive field and that of the gain control. In the LGN, we found normalization in magnocellular cells and cells receiving excitatory S-cone input but not in parvocellular cells or those receiving inhibitory S-cone input. We found normalization in all types of cortical neurons. Among cells that preferred achromatic modulation, or modulation along intermediate directions in color space (making them responsive to both achromatic and chromatic stimuli), normalization was driven by mechanisms tuned to a restricted range of directions in color space, close to achromatic. As a result, chromatic tuning varied with contrast. Among the relatively few cells that strongly preferred chromatic modulation, normalization was driven by mechanisms sensitive to modulation along all directions in color space, especially isoluminant. As a result, chromatic tuning changed little with contrast. To the extent that contrast invariant tuning is important in representing chromaticity, relatively few cortical neurons are involved.
\end{abstract}

Key words: vision; color; striate cortex; extrastriate; contrast; lateral geniculate

\section{Introduction}

Many aspects of the behavior of simple cells (and, by extension, complex cells) in the striate cortex can be explained by supposing that the receptive field sums contrast signals linearly, and that the summed signal is divided by another gain-controlling ("normalizing") signal that regulates the rate at which spikes are generated (Bonds, 1989; Geisler and Albrecht, 1992; Heeger, 1992; Carandini et al., 1997). This gain control is presumed driven by an activity-dependent signal accumulated from a pool of neurons tuned to a broad range of orientations and spatial frequencies. The uniformity of this normalizing signal across orientations and spatial frequencies preserves the tuning of a neuron in the face of contrast variations (Albrecht and Hamilton, 1982; Sclar and Freeman, 1982; Li and Creutzfeldt, 1984) and provides for a stable neural representation of the spatial attributes of an image. For a comparably stable neural representation of chromatic attributes, the normalizing signal would also need broad chromatic tuning.

The chromatic tuning of the normalizing signal is of considerable importance. Most neurons in V1 that respond well to achromatic stimuli also respond, although generally much less well, to chromatically modulated stimuli (Thorell et al., 1984; Lennie

Received Dec. 31, 2004; revised April 1, 2005; accepted April 6, 2005.

This work was supported by National Institutes of Health Grants EY04440 and EY13079 and Australian National Health and Medical Research Council Grant 211247 to S.G.S. We are grateful to N. Dhruv, J. Peirce, and C. Tailby for help during experiments and to M. Carandini, N. Graham, D. Heeger, G. Horwitz, J. Krauskopf, and C. Tailby for helpful advice.

Correspondence should be addressed to Dr. Samuel Solomon, University Laboratory of Physiology, University of Oxford, 0xford 0X1 3PT, UK. E-mail: samuel.solomon@physiol.ox.ac.uk.

D01:10.1523/JNEUROSCI.5316-04.2005

Copyright $\odot 2005$ Society for Neuroscience $\quad$ 0270-6474/05/254779-14\$15.00/0 et al., 1990; Johnson et al., 2001). Whether and how these weakly color-opponent neurons contribute to color vision is unclear. A useful approach to these questions is to explore the stability of the chromatic properties of a neuron while varying other attributes of the visual stimulus: an unstable signature, especially if it varied differently among cells, would suggest that a neuron was not a reliable source of information about color. In particular, we want to know whether chromatic properties are stable to variations in contrast. This would require either that the normalization signal be very broadly tuned in color space or that the neuron escape normalization altogether.

We do not know how the chromatic properties of neurons vary with contrast. We establish this here by measuring contrastresponse relationships with stimuli modulated along different directions in color space. We use these contrast-response curves to characterize the stability of chromatic tuning and to estimate the chromatic properties of the normalization pool.

\section{Materials and Methods}

Preparation and recording. Experiments were undertaken, as part of a larger series, on 22 adult male macaque monkeys (21 Macaca fascicularis and 1 Macaca radiata) weighing between 3.75 and $5.5 \mathrm{~kg}$. Each animal was anesthetized initially with ketamine hydrochloride (Vetalar; $10 \mathrm{mg}$ / $\mathrm{kg}$, i.m.). The saphenous veins were cannulated, and surgery was continued under thiopental sodium anesthesia. The monkey was intubated, the head was placed in a stereotaxic frame, and a craniotomy was made over the occipital cortex. Electrodes were attached to the skull to monitor the electroencephalogram (EEG) and to the forearms and legs to monitor the electrocardiogram (ECG). All procedures conformed to the guidelines approved by the New York University Animal Welfare Committee.

Postsurgical anesthesia was maintained by continuous infusion of sufentanil citrate $(4-12 \mu \mathrm{g} \cdot \mathrm{kg} \cdot \mathrm{h})$ in physiological solution (Nor- 
mosol-R; Abbott Laboratories, Abbott Park, IL) with added dextrose $(2.5 \%)$. Muscular paralysis was then induced and maintained by continuous infusion of vecuronium bromide $(100 \mathrm{mg} \cdot \mathrm{kg} \cdot \mathrm{h})$. The monkey was ventilated artificially so as to keep end-tidal $\mathrm{CO}_{2}$ near $33 \mathrm{mmHg}$. The EEG and ECG were monitored continuously, and at any sign of the anesthesia becoming less effective the dose of sufentanil citrate was increased. Temperature was monitored with a rectal probe and kept near $37^{\circ} \mathrm{C}$ with a heating blanket.

The pupils were dilated with atropine sulfate (typically to $7 \mathrm{~mm}$ ), and the corneas were protected with high-permeability contact lenses that remained in place for the duration of the experiment. No artificial pupils were used. Supplementary lenses (with power determined by ophthalmoscopy) were used to focus the eyes at a distance of $114 \mathrm{~cm}$. At the beginning of the experiment, and at regular intervals afterward, the positions of the foveas were mapped by reverse ophthalmoscopy. A small incision was made in the dura, and a guide tube containing the electrode (Ainsworth tungsten-in-glass or paralyene-coated tungsten; 1-5 M $\Omega$; FHC, Bowdoinham, ME) was positioned over this. The dura was covered with warm agar, and the craniotomy was sealed with dental acrylic. The analog signal from the electrode was amplified, filtered, and sampled at 11.025 or $22.05 \mathrm{kHz}$ by a dual processor Power Macintosh computer. Putative spikes were displayed on a monitor, and templates for discriminating spikes were constructed by averaging multiple traces. The timing of waveforms that matched the template was recorded with an accuracy of $0.1 \mathrm{~ms}$. Electrode tracks were reconstructed from the positions of the lesions made during the experiment as described previously (Solomon et al., 2004).

Visual stimuli. Visual stimuli were generated by the same computer that recorded spikes and were displayed, using commands to OpenGL, on a calibrated monitor (Sony G500 or EIZO T966), refreshed at $90 \mathrm{~Hz}$ and viewed from $114 \mathrm{~cm}$. Each grating was presented within a circular window. The remainder of the screen was held at the mean luminance of $\sim 50 \mathrm{~cd} / \mathrm{m}^{2}$ [Commission Internationale de l'Eclairage (1931); $x, \sim 0.30$; $y, 0.32]$. All stimuli were produced by spatiotemporal modulation around this point. These modulations can be represented in a threedimensional color space described previously (Derrington et al., 1984; Lennie et al., 1990). Along the L-M axis only the signals from $\mathrm{L}$ and $\mathrm{M}$ cones vary, in opposition, without variation in luminance. Along the orthogonal S-cone isolating axis there is no modulation of either the L or the $\mathrm{M}$ cones. The $\mathrm{L}-\mathrm{M}$ and $\mathrm{S}$ axes define a plane in which only chromaticity varies. Normal to this plane is the achromatic axis along which the signals from all three cone classes vary in proportion. Figure $1 \mathrm{~A}$ shows this color space. Full modulation along the $\mathrm{L}-\mathrm{M}$ axis gave cone contrasts of 0.08 for the $\mathrm{L}$ cones, 0.15 for the $\mathrm{M}$ cones, and 0.001 for the $\mathrm{S}$ cones. Full modulation along the $S$ axis produced contrasts of 0.85 for the $S$ cones and $<0.002$ for the $\mathrm{L}$ and $\mathrm{M}$ cones.

In all experiments the stimuli were grating patterns, or spatially uniform fields, defined by modulation along some vector through the white point in this space. The direction of the vector is defined by two angles: the elevation (the angle to the isoluminant plane, in which $90^{\circ}$ is the normal) and the azimuth (the angle within the isoluminant plane, in which $0^{\circ}$ represents $+\mathrm{L}-\mathrm{M}$ modulation and $270^{\circ}$ represents $+\mathrm{S}$ modulation). Gratings were modulated at five different contrasts along each of nine color vectors in this space. Where possible, we adjusted the range of contrasts along each color vector to include both the graded and saturating response levels. When making measurements, the stimuli in the set (one of which was always a blank screen) were presented in random order, each 10-20 times, in trials lasting $1 \mathrm{~s}$. Between trials the screen was blank (at the mean luminance) for $0.25 \mathrm{~s}$. From the train of impulses discharged during each stimulus presentation we extracted the mean rate and the amplitude of the Fourier component at the frequency of stimulation. Cells were identified as simple if the amplitude of the fundamental Fourier harmonic (F1) exceeded the elevation in the mean firing rate (Skottun et al., 1991), and for these cells we used the F1 as the measure of cell response.

The temporal frequency of the stimulus was usually $5 \mathrm{~Hz}$ but could be slightly higher in the lateral geniculate nucleus (LGN) (and occasionally lower in the cortex) in the cases when $5 \mathrm{~Hz}$ was far from optimal. The optimal spatial configuration was determined using a combination of
A

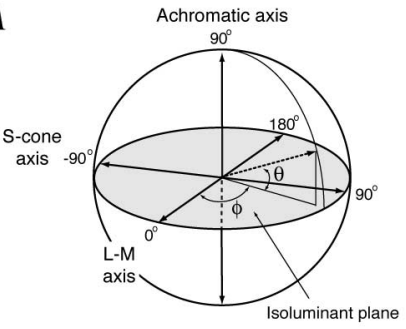

$B$

Normalization model

C

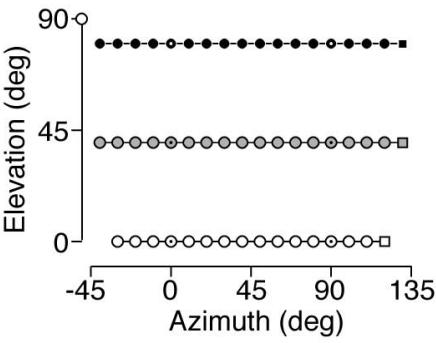

$D$

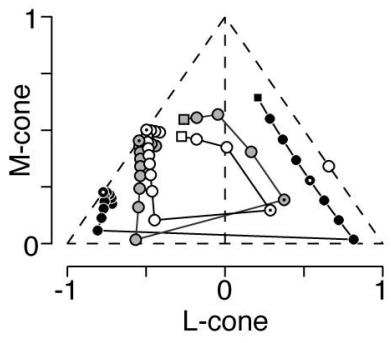

Figure 1. A, Color space used to represent stimuli. It is defined by an L-M axis along which the signals of the $L$ and $M$ cones covary to keep their sum constant, an S-cone isolating axis, and an achromatic axis where the signals of the three cone classes vary in proportion. The L-M- and $S$-cone axes define an isoluminant plane, where chromaticity varies without a change in luminance. Stimuli are specified by their azimuth in the isoluminant plane $(\phi)$ and their elevation from the isoluminant plane $(\theta)$. $\boldsymbol{B}$, Model cortical receptive field incorporating normalization pool [following Carandini et al. (1997)]. The LRF computes a weighted linear sum over local contrast and chromaticity. This signal is then divided by a normalization signal with a chromatic signature that can differ substantially from that of the receptive field. $\boldsymbol{C}, \boldsymbol{D}$, Relationship between the preferred azimuth and elevation of the LRF and the weights assigned to signals from different classes of cones. Each combination of azimuth and elevation represented by a point in $\boldsymbol{C}$ has a counterpart point in $\boldsymbol{D}$ that shows the weights on the three classes of cones. Each connected sets of points characterizes a different preferred elevation: $0^{\circ}$ (open symbols), $40^{\circ}$ (gray symbols), and $80^{\circ}$ (filled symbols) at a range of azimuths. The isolated open symbol in $\boldsymbol{D}$ shows the cone weights associated with a preferred elevation of $90^{\circ}$. Symbols containing small dots represent LRFs with preferred azimuths of 0 and $90^{\circ}$. Conventions used in $C: L$ - and $M$-cone inputs of the opposite sign are represented with negative L-cone weights; the strength of the S-cone input is represented by the distance inside the diagonal. In this and the following figures, degree is abbreviated as "deg."

quantitative measurements and iterative search by the experimenter. When the neuron responded to achromatic gratings, these were used to determine the optimum orientation and spatial frequency; we know that these parameters will be the optimal, or near optimal, for chromatic gratings (Johnson et al., 2001; Solomon et al., 2004). In contrast, we have shown recently (Solomon et al., 2004) that the relative sensitivity of neurons to different colors often depends on the size of the stimulus used. We minimized the effect of surround suppression by using grating patches of optimum size (established using achromatic gratings). For LGN neurons, the grating patch was always much larger than the receptive field. Almost all of the cortical cells that we studied had receptive fields within $5^{\circ}$ of the fovea, and most were within the central $2^{\circ}$; receptive fields in the LGN lay between 5 and $25^{\circ}$ from the fovea.

A normalization model generalized for chromatic signals. A broad range of visual characteristics of a V1 neuron can be explained by supposing that the cell computes a weighted linear sum of local contrast over its receptive field, with the resultant output being divided by a separate neural measure of stimulus energy. For achromatic stimuli this model, explained fully by Carandini et al. (1997) and illustrated graphically in Figure $1 B$, predicts the following response amplitude:

$$
R_{(\mathrm{c})}=R_{\max }\left[\frac{c}{\sqrt{\sigma^{2}+c^{2}}}\right]^{n},
$$

where $c$ is contrast, the exponent $n$ is an output nonlinearity, $R_{\max }$ is an amplitude scaling factor, and $\sigma$ is the sensitivity of the pool. To generalize this model to chromatic signals, we need to specify contrast in the full 
color space of Figure $1 \mathrm{~A}$. We also need to allow the linear receptive field (LRF) (the numerator) to take a different measure of contrast than the normalization pool (the denominator). For chromatic signals the equation therefore becomes:

$$
R_{(\lambda)}=R_{\max }\left[\frac{S_{(\lambda)}}{\sqrt{\sigma^{2}+N_{(\lambda)}^{2}}}\right]^{n},
$$

where $S_{(\lambda)}$ is the activity of the LRF and $N_{(\lambda)}$ is the activity of the normalization pool. Here, $\lambda$ denotes not wavelength but any vector in the threedimensional color space of Figure $1 A$. The signals $S_{(\lambda)}$ and $N_{(\lambda)}$ are each the product of the strength of the stimulus (modulation depth) and sensitivity to the stimulus. We use a quasilinear model of cone summation to estimate $S_{(\lambda)}$ (see below, Estimating cone inputs to receptive fields); for the normalization pool we will construct an estimate of chromatic contrast energy, below.

This general model of normalization predicts response amplitude but makes no assumptions about the biophysical mechanisms that underlie it. For quasilinear cells that give modulated responses we also have measurements of response phase and can use these to examine predictions of a particular biophysical implementation of the normalization model (Carandini and Heeger, 1994; Carandini et al., 1997) that derives linked changes in response amplitude and phase from changes in membrane conductance. Briefly, response amplitude is given by Equation 2, but with the semi-saturation constant $(\sigma)$ calculated as follows:

$$
\sigma=\frac{1+\left(2 \pi f \tau_{0}\right)^{2}}{\left(\tau_{0} / \tau_{1}\right)^{2}-1} .
$$

$\sigma$ is thus determined by the temporal frequency $(f)$ and two time constants $\left(\tau_{0}\right.$ and $\left.\tau_{1}\right)$. The actual values of the time constants are unimportant, for they are used only to link response amplitude to response phase, given by the following:

$$
\operatorname{Phase}_{(\lambda)}=P_{(\lambda)}-\frac{\operatorname{atan}\left(2 \pi f \tau_{0}\right)}{\sqrt{1+N_{(\lambda)}{ }^{2} \times\left(\left(\tau_{0} / \tau_{1}\right)^{2}-1\right)}},
$$

where $P_{(\lambda)}$ is the phase of response from the LRF.

Changing the color vector along which stimuli are modulated changes the relative amplitudes of L-, M-, and S-cone signals. How the gain control combines these signals will therefore determine its susceptibility to stimulus modulations along different vectors. Given the coloropponent transformations that occur in the retina, it seems unlikely that contrast signals from the different classes of cones are available separately to the cortex, but rather in linear combinations that reflect the properties of three second-stage chromatic mechanisms. We have assumed provisionally that these are the cardinal mechanisms identified psychophysically (Krauskopf et al., 1982) and that the signal from each is half-wave rectified (Chen et al., 2000b) then squared and summed in spatiotemporal quadrature (Heeger, 1992). The three resulting mechanisms, denoted $I_{\mathrm{LUM}}, I_{\mathrm{LM}}$, and $I_{\mathrm{S}}$, combine cone signals as follows:

$$
\begin{aligned}
I_{\mathrm{LUM}} & =[1.94 \times \mathrm{L}+\mathrm{M}]^{2} \\
I_{\mathrm{LM}} & =[\mathrm{L}-\mathrm{M}]^{2} \\
I_{\mathrm{S}} & =[\mathrm{S}-0.5 \times(\mathrm{L}+\mathrm{M})]^{2},
\end{aligned}
$$

where 1.94 is the relative weight of L- and M-cone signals in the luminosity function $\left(V_{\lambda}\right)$. Each mechanism was assumed to generate unit response to the maximum realizable modulation along its preferred axis. The chromatic signature of the normalizing signal is determined by a weighted combination of the outputs from these mechanisms:

$$
N_{(\lambda)}=\left[w_{\mathrm{LUM}} \times I_{\mathrm{LUM}}+w_{\mathrm{LM}} \times I_{\mathrm{LM}}+w_{\mathrm{S}} \times I_{\mathrm{S}}\right]^{0.5},
$$

where $w_{\text {LUM }}$, etc., is the weight of the relevant mechanism. The three weights define a vector in the color space with preferred elevation $\theta_{\mathrm{N}}$ and preferred azimuth $\phi_{\mathrm{N}}$; these were estimated by the fitting routine (see below, Model fitting). When all three mechanisms have equal weight in the normalization pool, the pool is equally sensitive to modulation along all directions in color space; when one or two mechanisms dominate, the distribution of sensitivity in the pool is correspondingly narrowed.

To display contrast-response functions, we wanted a contrast metric that incorporated cone contrast and could represent modulation depth along any color direction. For purposes of representation, we therefore compute the contrast as follows:

$$
c=\left[w_{\mathrm{L}} L_{2}+w_{\mathrm{M}} \mathrm{M}^{2}+w_{\mathrm{S}} \mathrm{S}^{2}\right]^{0.5},
$$

where $L$ is the $\mathrm{L}$-cone contrast in the stimulus and $w_{\mathrm{L}}$ is the weight attached to the L-cone signal, and similarly for $\mathrm{M}$ and $\mathrm{S}$ cones. We call this measure the wRMS contrast, because of its similarity to root-meansquare contrast. A reasonable estimate of the weights would reflect the relative numbers of the different classes of cones in the macaque retina. We do not know this precisely for $\mathrm{L}$ and $\mathrm{M}$ cones and have taken it to be the weight of $\mathrm{L}$ - and $\mathrm{M}$-cone signals in the human luminosity function $\left(V_{\lambda} ; w_{\mathrm{L}}=1.94 \times w_{\mathrm{M}}\right)$; we therefore weigh signals from the cone classes as follows:

$$
w_{\mathrm{L}}=0.59 ; w_{\mathrm{M}}=0.31 ; w_{\mathrm{S}}=0.1 \text {. }
$$

Estimating cone inputs to receptive fields. In Results, we analyze the properties of two mechanisms that might have different chromatic signatures: an LRF and the normalizing gain control. To obtain the signature of the LRF and therefore the weights it assigned to inputs from different cone classes, we assumed that responses of near-threshold amplitude do not consequentially activate the normalization pool. We measured responses to modulation along each of nine directions in color space, and fitted a simple model. We first fitted Equation 2 separately to the contrast-response function (five contrasts) obtained for each direction of modulation, and then from each curve estimated the contrast required for a small criterion response (the larger of two impulses $\cdot \mathrm{s}^{-1}$ or 2 SDs above the spontaneous rate). For any direction of modulation for which the response was too weak to be fitted, we set sensitivity to zero.

If a cell combines cone signals linearly and responds in proportion to this combined signal, then the amplitude of response to any color direction (vector) is given by the dot product of the stimulus vector and the vector that describes the preferred color direction of the cell, such that:

$$
R=K\left(\sin \theta \sin \theta_{\mathrm{m}}+\cos \theta \cos \theta_{\mathrm{m}} \cos \left(\phi-\phi_{\mathrm{m}}\right),\right.
$$

where $R$ is sensitivity (the reciprocal of contrast at threshold), $K$ is a scale factor, $\theta$ and $\phi$ are the elevation and azimuth of the stimulus vector, and $\theta_{\mathrm{m}}$ and $\phi_{\mathrm{m}}$ are the elevation and azimuth of the preferred color direction vector of the cell. The sign of the response to a particular direction of modulation is given by the response phase, which we obtained from the response to the highest contrast tested. For a complex cell, this information is unavailable, and so we used a full-wave rectified version of the linear model for those cells. Some cells show sharper tuning than is predicted by the linear model of Equation 9. Their behavior is well captured by supposing that the signal passes through an expansive nonlinearity (Kiper et al., 1997; De Valois et al., 2000). We therefore assumed this for all cells, constraining the exponent of the expansive nonlinearity to be between 1 and 5 .

Although the preferred elevation and azimuth provide a convenient indication of the chromatic signature of a cell, it is more fundamentally represented by the relative weights the cell assigns to the modulated signals from each of the cone classes. These can be derived from the preferred color directions of Equation 9 (Lennie et al., 1990).

Model fitting. Although our normalization model makes clear predictions, it could be hard to characterize in some cells: (1) those in which the chromatic signature of the normalization pool is exactly the same as that of the LRF (equivalent to a static compressive nonlinearity) and (2) those in which the normalization pool is so weak, or is tuned to directions in color space that elicit no response from the LRF, that it brings about no contrast-dependent saturation of response. We therefore compared the performance of the full normalization model with that of two variant cases (a static compressive nonlinearity and a simple linear model).

For each cell, we fitted the variant forms of Equation 2, including in the data set the responses to every color direction in which response to the highest contrast was significantly above baseline $(p<0.05$; Student's $t$ 
test). Each fit minimized the $\chi^{2}$ error between the prediction and the data:

$$
\chi^{2}=\Sigma_{\mathrm{i}} \frac{\left(e_{\mathrm{i}}-o_{\mathrm{i}}\right)^{2}}{\sigma_{i}^{2}},
$$

where $e_{\mathrm{i}}$ is the model prediction for the $i$ th response, $o_{\mathrm{i}}$ is the mean of the observed response, and $\sigma_{i}^{2}$ is the variance of the observed response. The error can be calculated just for response amplitude or for both amplitude and phase in the complex plane. To avoid placing too much emphasis on small responses, we gave $\sigma^{2}$ a lower bound of 1 . When the lower bound is in effect (as was the case for 70 of 205 cells), the error term is not a true $\chi^{2}$ value, although its relative size across models remains informative. The fitting function (Matlab version 6.5; Mathworks, Natick, MA) minimized the $\chi^{2}$ using a Levenberg-Marquet optimization routine. Because different variants of the model had different numbers of parameters, in comparing the quality of fits we used a $\chi^{2}$ measure incorporating the degrees of freedom in the model $\left(\chi^{2}\right.$ norm $\left.=\chi^{2} / \mathrm{df}\right)$. Degrees of freedom also depend on the number of color directions for which responses were obtained. The median number of observations per cell was 40 (responses to eight color directions at five contrasts), and thus $\mathrm{df}=27$ for the normalization model. To understand better the power of the model, we also determined the percentage of variance it left unexplained (Carandini et al., 1997). We calculated the response variance as follows:

$$
V_{\text {resp }}=1 / N \Sigma_{\mathrm{n}}\left(R-R_{\mathrm{s}}\right)^{2},
$$

where $R$ is the mean response across trials for each stimulus and $R_{\mathrm{s}}$ is the mean response across stimuli. The difference between the model prediction for each stimulus $\left(R_{\mathrm{m}}\right)$ and the response $(R)$ of the cell can be determined by substituting $R_{\mathrm{m}}$ for $R_{\mathrm{s}}$ in Equation 11 . We call the resultant value $V_{\text {model }}$. The percentage of variance left unexplained is $100 \times$ $V_{\text {model }} / V_{\text {resp. }}$. This measure expresses the relative capacity of each model to explain the data and depends on both the quality of the model and the variance (range) of observed responses. The range of response amplitudes of V2 neurons was less than those of V1 neurons, principally because the former were more often saturated (Levitt et al., 1994). This reduced range led to a greater unexplained variance for $\mathrm{V} 2$ neurons than V1 (median, 6.8 vs $2.8 \%$ ), although the goodness of fit was similar in the two areas (median $\chi_{\text {norm }}^{2}=0.102$ in V2 and 0.100 in V1).

\section{Results}

We report on responses obtained from single neurons in the LGN $(n=46), \mathrm{V} 1(n=116)$, or V2 $(n=52)$ to temporally modulated uniform fields, or drifting gratings of optimal spatial frequency, orientation, and direction of movement for the neuron under study. Our sample is biased. Especially early in this study, we were more likely to collect data from neurons that responded to isoluminant modulation. Our observations and analysis will show that the overall chromatic tuning of a neuron results from the interplay of two mechanisms that often have different chromatic signatures: an LRF and a normalizing gain control. It is helpful in organizing the analysis of normalization to have cells characterized by the chromatic properties of the LRFs, and we do this first. We go on to characterize the chromatic signatures of the normalizing gain controls in individual cells and then show how the properties of these gain controls bring about contrast-dependent changes in the chromatic tuning of most cells. Finally, we show that the expression of the gain controls in cortical neurons is not simply inherited from the LGN.

\section{Cone inputs to the LRF}

The sensitivity of a neuron to stimulus modulation along different color directions is determined by the way in which it combines cone signals. For a neuron that combines the signals from the three classes of cones linearly, the weights it attaches to signals
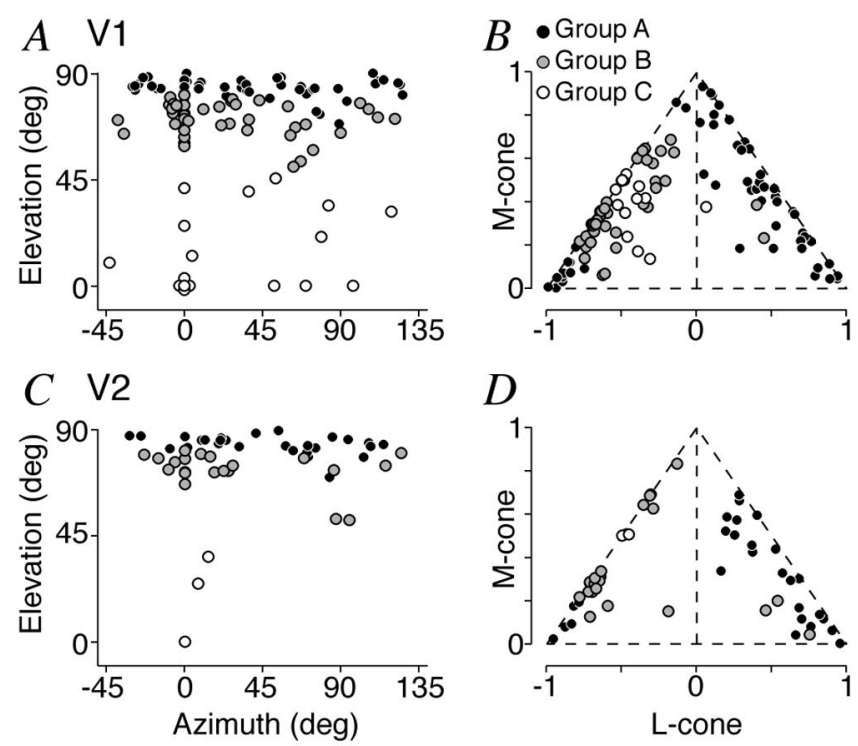

Figure 2. Chromatic signatures of receptive fields in V1 and V2. $A, C$, Distributions of the preferred directions in the color space of Figure $1 A . \boldsymbol{B}, \boldsymbol{D}$, Distributions of the relative weights attached to inputs from each cone class. Here and in subsequent figures, the different symbols distinguish the three groups of cells discussed in the text: group A, cells that preferred achromatic modulation (filled symbols); group $B$, cells that preferred modulation at intermediate elevations (gray symbols); group C, cells that preferred isoluminant modulation (open symbols). In $\boldsymbol{A}$ and $\boldsymbol{C}$, the preferred azimuths and elevations have been reflected into a reduced space that does not distinguish cells with complementary signatures. $\ln \boldsymbol{B}$ and $\boldsymbol{D}$, the true signs of cone inputs are unknown, so cells with L- and M-cone inputs of the opposite sign are shown with negative $L$-cone weights. The strength of $S$-cone input is represented by distance inside the diagonal.

from each are expressed as a characteristic preferred direction (azimuth, elevation) in color space and can be derived directly from measurements of its responses to modulation along different directions [see Materials and Methods and examples in Derrington et al. (1984), Lennie et al. (1990), and Solomon et al. (2004)]. Normalization is presumed weak at low contrast, so by working with near-threshold responses to stimulus modulation along different color directions, we can derive the preferred direction of the LRF in color space and the signs and weights of its cone inputs. The quasilinear model we have used captures the measurements well: the median unexplained variance in fits (Carandini et al., 1997) was 7.0\% in V1 and 5.0\% in V2.

Figure 2 shows for our V1 and V2 cells the distributions of the preferred directions in color space (Fig. $2 A, C$ ) and the corresponding distributions of cone weights (Fig. $2 B, D$ ). The signs of cone inputs cannot be recovered from our measurements (Johnson et al., 2001), so we have adopted the convention that cells with L- and M-cone inputs of the opposite sign are shown with negative L-cone weights. The distance from the diagonal represents the strength of S-cone input to the receptive field. The representations of the chromatic signature of a cell by the preferred direction (azimuth, elevation) and distribution of cone weights (LMS) are equivalent, but in moving between them it is important to note that the color space in Figure $1 A$ is normalized to the maximum attainable modulation along each of the cardinal axes. Full modulation along the achromatic axis generates greater cone contrasts than full modulation along isoluminant color directions. The upshot is that at low elevations a large change in elevation represents a small change in the weights assigned to different cone inputs, whereas at high elevations a small change in elevation can represent a large change in weights. This is illustrated in Figure 1, $C$ and $D$. 

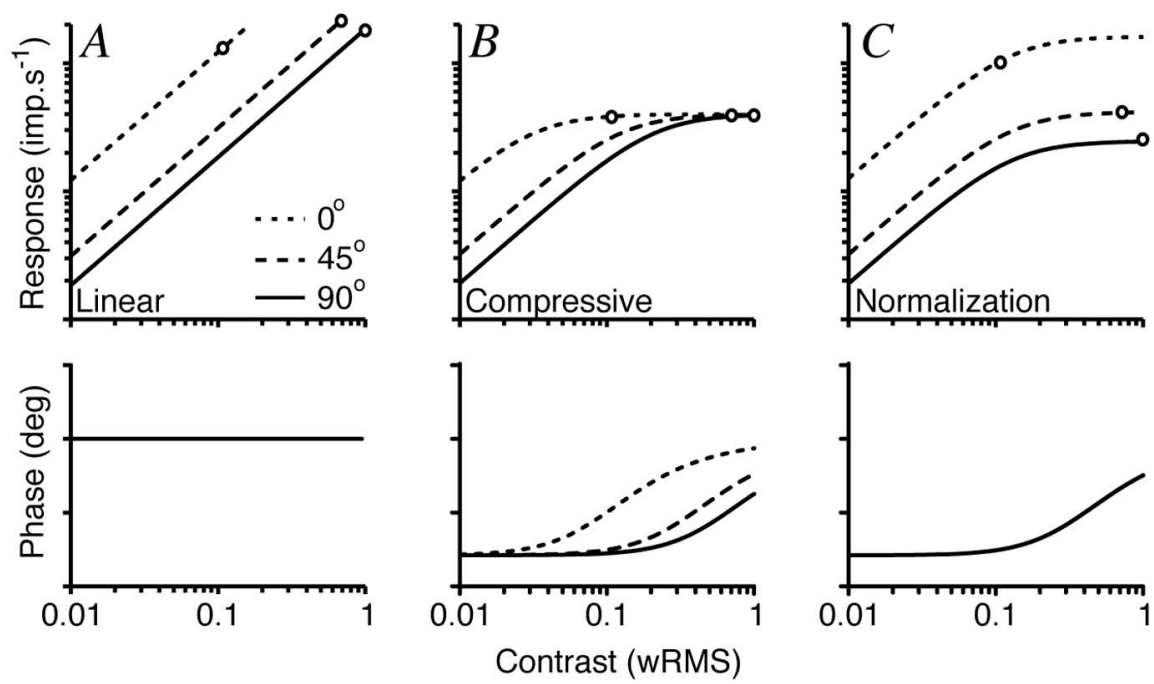

Figure 3. Three models of chromatic response regulation in a simple cell with a preferred elevation of $50^{\circ}$ and a preferred azimuth of $0^{\circ}$. Each pair of panels shows response magnitude (top) and response phase (bottom) as a function of the wRMS contrast in the stimulus (see Materials and Methods for derivation). Responses are shown for three different directions of modulation in the L-M/achromatic plane: isoluminant ( $0^{\circ}$ elevation), achromatic ( $90^{\circ}$ elevation), and intermediate ( $45^{\circ}$ elevation). $\boldsymbol{A}$, Linear: response increases in proportion to contrast and phase is independent of contrast. $\boldsymbol{B}$, Compressive nonlinearity: amplitude and phase depend only on the capacity of the stimulus to drive the receptive field. Amplitude asymptotes at the same level for all color directions, and phase begins to advance at different contrast levels. C, Normalization by a mechanism that is equally sensitive to modulation along all directions in color space: the shapes of contrast-amplitude and contrast-phase curves are determined in contrast, and not the effectiveness of a particular stimulus for the LRF. Response amplitudes asymptote at different values, and response phases are identical. The circles indicate the maximum contrast achievable along each color direction on our monitor. In this and the following figures, impulses is abbreviated as "imp."

To help organize the analysis of normalization, we have placed cells into three informal groups, based on the chromatic signatures of their LRFs. One group of cells (18 in V1 and 3 in V2), which we will call "group C," preferred chromatic modulation and had elevations within $50^{\circ}$ of the isoluminant plane in the color space of Figure $1 \mathrm{~A}$. Most of these neurons responded to temporal modulation of a spatially uniform field as well as they did to gratings (15 in V1 and 2 in V2) and were insensitive to orientation (16 in V1 and 2 in V2) (Lennie et al., 1990; Johnson et al., 2001; Solomon et al., 2004); their firing rates were modulated at the frequency of stimulation (except for one cell in V1 and one cell in V2). A second group of cells (42 in V1 and 22 in V2), which we will call "group B," received weakly opponent cone inputs with none providing $>80 \%$ of the total input; they had elevations within $80^{\circ}$ of the isoluminant plane. If we assumed linearity of signal summation, a neuron with a preferred elevation of $80^{\circ}$ would respond slightly better to achromatic gratings of contrast 0.2 than to isoluminant gratings of the highest contrast we could produce. The third group, which we will call "group A," preferred achromatic modulation and contained all the remaining cells (56 in V1 and 27 in V2). These groupings are arbitrary but convenient; they are broadly similar to the groups we (Solomon et al., 2004) and Johnson et al. $(2001,2004)$ have used previously, but because the current assignment is based on threshold responses, and because many neurons gave saturated responses to achromatic contrasts as low as 0.2 , group A contains relatively more neurons than the "luminance-preferring" groups of previous work.

We were able to recover the laminar locations of $52 \mathrm{~V} 1$ cells. As noted previously (Lennie et al., 1990; Johnson et al., 2001), most of the group C cells (13 of 14) were found in layers IVC $\beta, \mathrm{V}$, and VI, and a large proportion (6 of 10) of the neurons in layers $2 / 3$ were weakly opponent group B cells.
Possible expressions of normalization

It is helpful to visualize the impact of normalization by plotting responses as a function of stimulus contrast. Figure $3 A-C$ shows theoretical contrast-amplitude functions (top panels) and contrast-phase functions (bottom panels) but derived from different assumptions about the properties of the normalization pool. In each panel the three curves represent the behavior of a simple cell (preferred direction in the color space of Fig. $1 \mathrm{~A}$; elevation, $50^{\circ}$; azimuth, $0^{\circ}$ ) driven by modulation along three color vectors: the L-M axis (elevation, $0^{\circ}$; azimuth, $0^{\circ}$ ), the achromatic $\mathrm{L}+\mathrm{M}+\mathrm{S}$ axis (elevation, $90^{\circ}$ ), and the vector midway between these axes (elevation, $45^{\circ}$; azimuth, $0^{\circ}$ ). Responses are plotted against the weighted RMS cone contrast (Eq. 7) in each stimulus. We use this contrast metric throughout to make comparable the contrast-response curves obtained with stimuli modulated along different color directions.

Figure $3 A$ illustrates contrast-response functions we would expect from a cell that possesses no normalization pool: the signal from an LRF is passed through an expansive output nonlinearity, and the phase is independent of contrast. [The preferred elevation of $50^{\circ}$, despite its high responsivity to isoluminant stimuli, reflects the scaling of the color space in which elevation and azimuth are calculated (Fig. 1C,D). ] Figure $3 B$ shows the behavior expected if the normalization pool has the same spectral sensitivity as the LRF (i.e., $N_{(\lambda)}=S_{(\lambda)}$ in Eqs. 2-4). In such a case all curves become asymptotic at the same level of response, as if the response of the LRF had been passed through a (static) compressive nonlinearity. For all color directions, response phase advances with increasing contrast. The contrast at which phase begins to advance is determined by the chromatic signature of the normalization pool. In this case the normalization pool has the same chromatic signature as the LRF, so for color directions to which the neuron is more sensitive phase begins to advance at lower contrasts.

Figure $3 C$ shows the contrast-response functions expected when the chromatic signature of the normalization pool differs from that of the LRF. In this example the normalization pool is equally sensitive in all color directions. The relative heights of the three curves are determined by the sensitivity of the LRF, but the contrasts at which curves roll over are determined by the activity of the normalization pool. The shapes of curves will therefore depend on the spectral composition of inputs to the pool, and responses to modulation along different color vectors will generally become asymptotic at different amplitudes. The shapes of the contrast-phase curves will reflect the strength of the normalization signal but not the sensitivity of the LRF.

Some neurons in V1 (particularly those that are most sensitive to chromatic modulation) yield contrast-response curves that show little saturation, making it hard to characterize any normalization. In other neurons (particularly those that prefer achromatic stimuli) the normalization pool is likely to have the same chromatic signature as the LRF, making it hard to distinguish normalization from a static nonlinearity. In what follows we have 

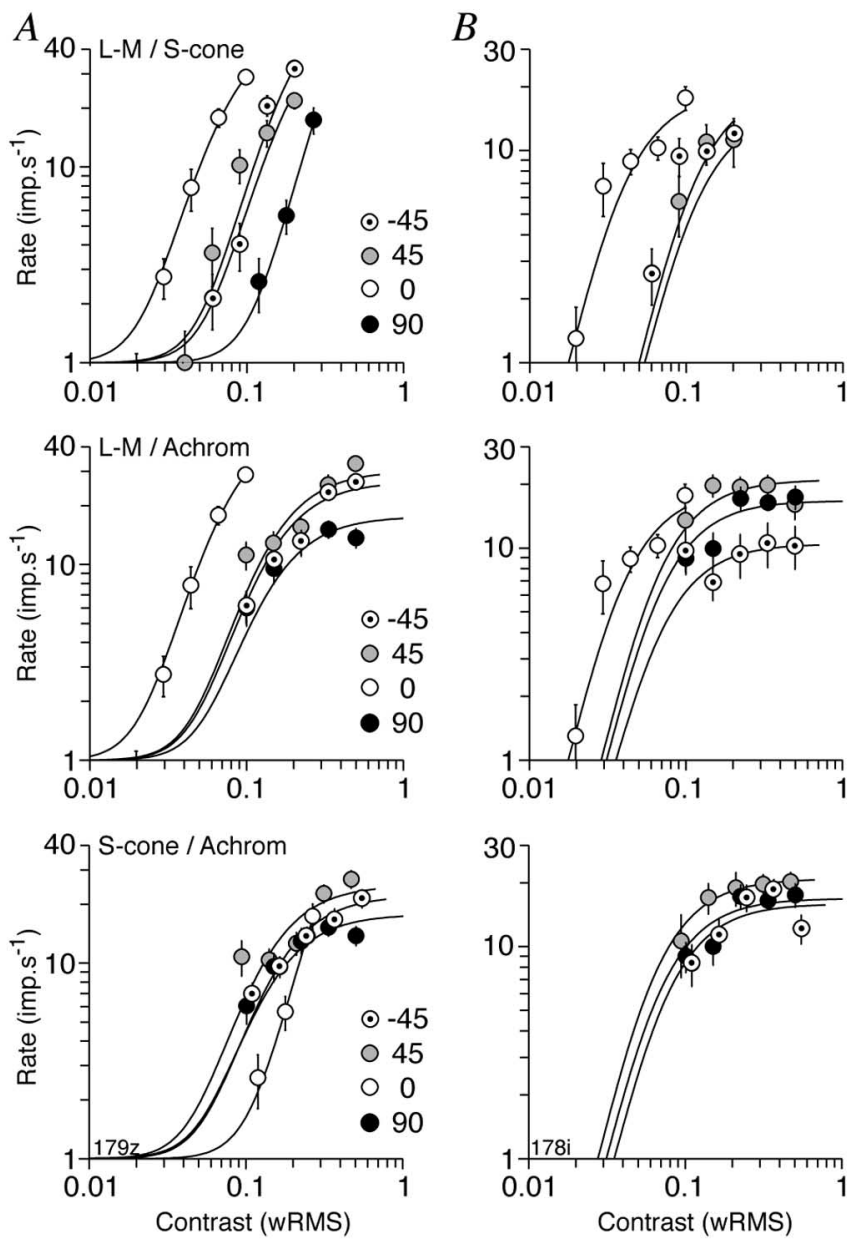

Figure 4. Normalization in complex cells. $A$, Sets of contrast-response curves for a weakly opponent cell (group B) in V1. Top, Mean rate to stimuli modulated along the identified directions in the isoluminant plane (elevation, $0^{\circ}$ ). Middle, Mean rate to stimuli modulated along the identified elevations in the plane formed by the $L-M$ axis (azimuth, $0^{\circ}$ ) and the achromatic axis. Bottom, Mean rate to stimuli modulated along the identified elevations in the plane formed by the $S$-cone axis (azimuth, $90^{\circ}$ ) and the achromatic axis. $\boldsymbol{B}$, As for $A$, except for a weakly opponent (group B) complex cell in V2. Missing curves in the top and bottom panels reflect the absence of a response to modulation along the $S$-cone axis. Solid lines are the predictions of the normalization model described in Materials and Methods, fit to the mean response rates obtained. Model and stimulus parameters: for $\boldsymbol{A}, \sigma=0.11, n=3.9, \theta_{\mathrm{N}}=88.1^{\circ}, \phi_{\mathrm{N}}=9.0^{\circ}, 1.0$ cycles - degree ${ }^{-1}, 5.3 \mathrm{~Hz}$; for $\boldsymbol{B}, \sigma=0.05, n=4.6, \theta_{\mathrm{N}}=88.4^{\circ}, \phi_{\mathrm{N}}=0.0^{\circ}, 3.0$ cycles $\cdot$ degree $^{-1}, 4.8 \mathrm{~Hz}$. Individual parameters estimated the height of each curve. Error bars are \pm 1 SEM from 20 repetitions. In this and the following figures, "Achrom" refers to the achromatic axis.

therefore been careful to compare the predictions of the normalization model against those of the two simpler models represented in Figure 3, $A$ and $B$.

\section{Effects of contrast on responses to chromatic modulation}

Figure 4 shows the responses of two complex cells to stimulus modulation along various color directions. In this and subsequent figures, we plot separately responses to modulation in each plane of color space. The top panels of Figure 4 show responses to modulation within the isoluminant plane (elevation, $0^{\circ}$ ), and angles refer to the azimuth $(\phi)$ of modulation. The middle panels show responses to modulations within the plane formed by the $\mathrm{L}-\mathrm{M}$ axis and achromatic axis; these all have an azimuth of $0^{\circ}$, and angles refer to the elevation $(\theta)$ of modulation. The bottom panels show responses to modulations in the plane formed by the
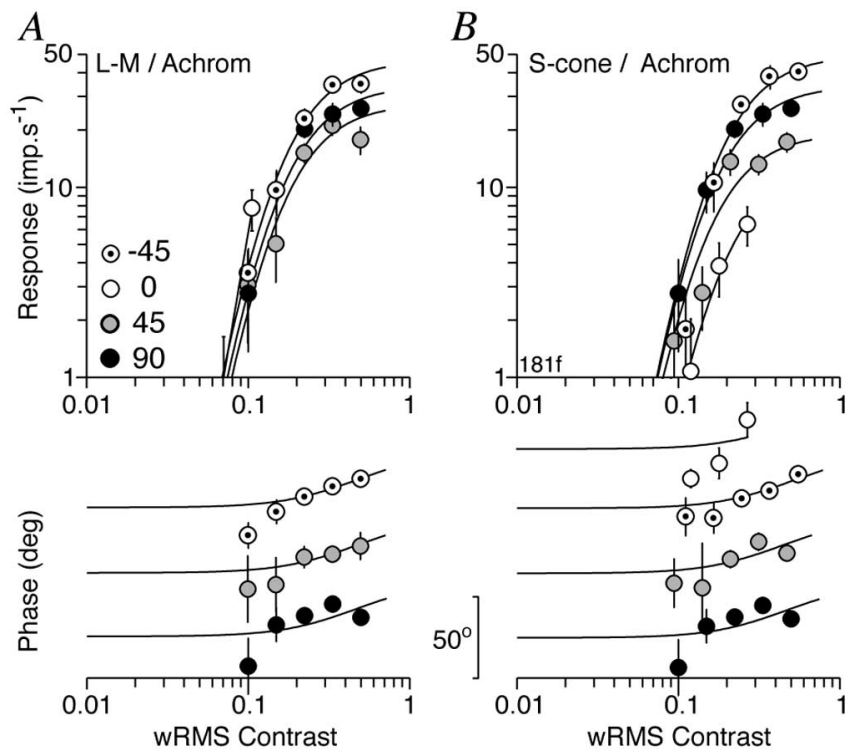

Figure 5. Normalization in a simple cell. Sets of contrast-response curves for a weakly opponent cell (group B) in V1. A, Responses are shown for stimuli modulated along the identified elevations in the L-M/achromatic plane. The top and bottom panels show, respectively, response amplitude and phase at the frequency of modulation. The phase curves have been spaced vertically for clarity. $\boldsymbol{B}$, Same as $\boldsymbol{A}$, except the plane of modulation was S-cone/achromatic. Solid lines are the predictions of the normalization model described in Materials and Methods, fit to response amplitude and phase in the complex plane. Model and stimulus parameters: $t_{0}=25.2 \mathrm{~ms} ; t_{1}=6.7 ; n=5.0 ; \theta_{\mathrm{N}}=87.0^{\circ} ; \phi_{\mathrm{N}}=90.0^{\circ} ; 1.4$ cycles $\cdot$ degree $^{-1}$; $5.3 \mathrm{~Hz}$. Individual parameters estimated the height of each curve. Error bars are \pm 1 SEM from 13 repetitions.

S-cone axis and the achromatic axis; the azimuth is $90^{\circ}$, and angles refer to the elevation. Responses to modulation in a particular direction are omitted if the maximal response did not reach criterion levels.

The cell in Figure $4 A$ revealed normalization by a mechanism with a spectral signature that differed from that of the LRF. This is particularly clear in the comparison of the three contrast-response curves in the $\mathrm{L}-\mathrm{M} /$ achromatic plane (Fig. $4 \mathrm{~A}$, middle) Responses to achromatic gratings saturated rapidly with increasing contrast, but at one-half the height reached for the intermediate vector. Responses to isoluminant L-M gratings did not saturate. Normalization is less evident in the responses to modulation within the plane formed by the S-cone and achromatic axis (Fig. $4 A$, bottom). Figure $4 B$ shows sets of contrastresponse curves for a V2 complex cell. As for the V1 neuron, responses to isoluminant modulation showed little or no saturation, whereas responses to modulation along other directions saturated at different amplitudes.

The top panels in Figure 5, $A$ and $B$, show, in the same format as Figure 4, curves derived from the modulated responses of a V1 simple cell. As for the complex cells in Figure 4, the responses to modulation along different color vectors saturate at different amplitudes. The bottom panels in Figure 5 show counterpart plots of response phase. Phase advances as contrast is increased, and in the same way for every direction of modulation, despite the large difference in response amplitudes.

Figure 6 shows, in the same format as Figures 4 and 5, contrast-response curves for three neurons that responded best to chromatically modulated stimuli. Curves for group $\mathrm{C}$ cells were generally more linear than those of other neurons (e.g., Fig. 6A), and in only approximately one-half were there clear signs of saturation (e.g., Fig. 6B,C). Of the eight color-preferring V1 neu- 
A

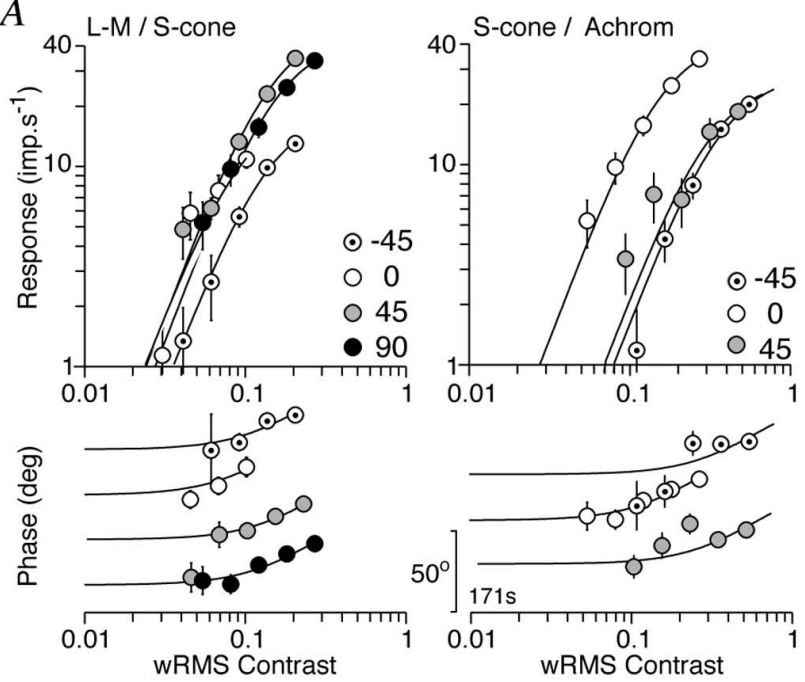

$B$
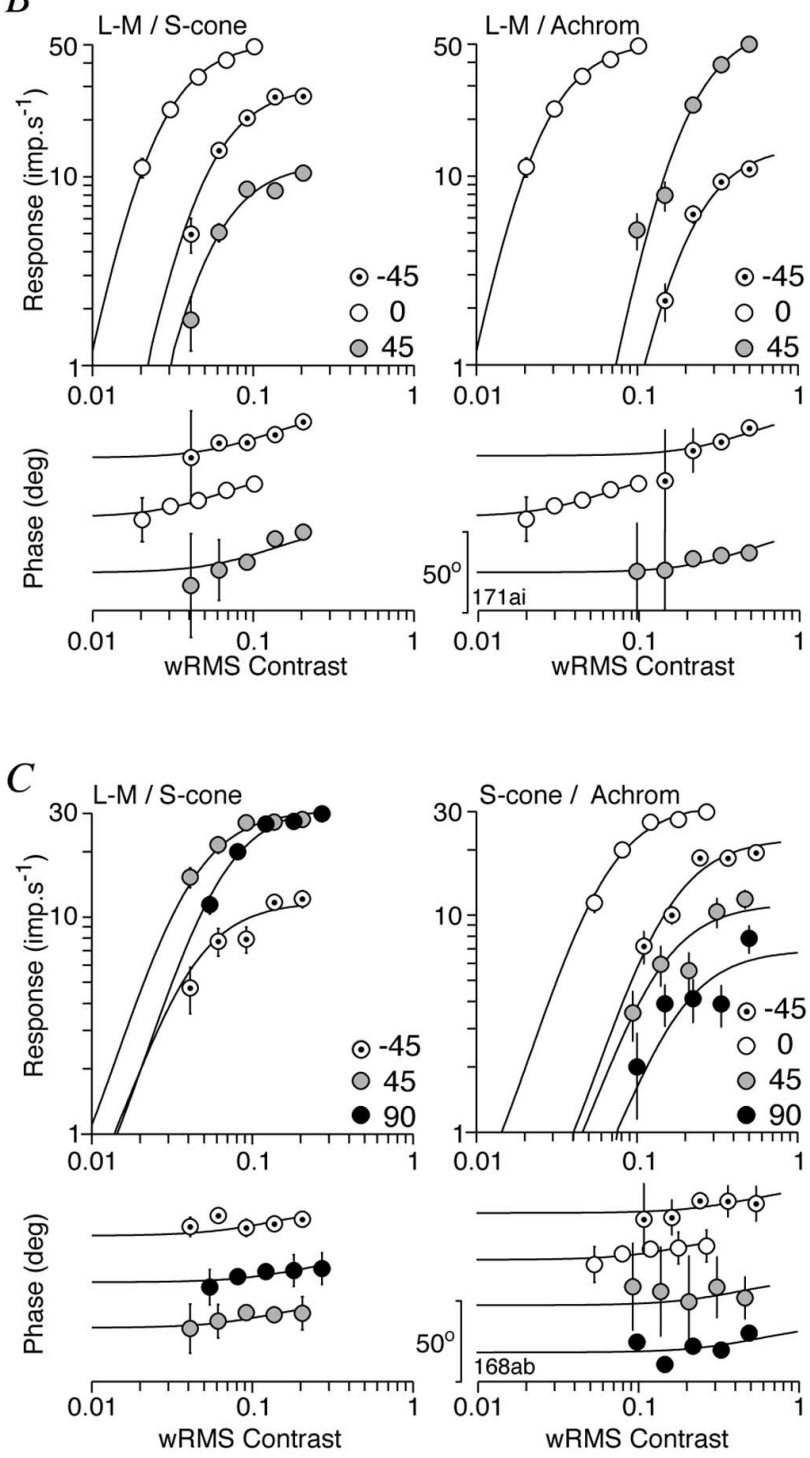

Figure 6. Normalization in color-preferring cells (group C) in V1. A, Sets of contrast-response curves for a cell that preferred S-cone modulation. Responses are shown for stimuli modulated along the identified azimuths in the isoluminant plane (left) and the identified elevations in the plane formed by the $\mathrm{S}$-cone and achromatic axes (right). The top and bottom rons that responded strongly to S-cone isolating modulation, only two gave saturated responses. Figure $6 C$ is an example. Among neurons that showed saturation, responses to modulation along different color directions usually saturated at different amplitudes, implying, as for the simple and complex cells shown in Figures 4 and 5, that the spectral sensitivity of the normalization pool differed from that of the LRF.

The generally more linear behavior of group $\mathrm{C}$ neurons is expressed in lower exponents of their contrast-response curves (mean of 2.6 vs 3.1 for group B cells and 4.1 for group A cells). This does not result from responses of group C cells being smaller; responses are, in fact, among the strongest: average maximum response of 38.0 impulses $\cdot \mathrm{s}^{-1}$ versus 36.5 impulses $\cdot \mathrm{s}^{-1}$ for other cells. Despite their more linear behavior, many group $\mathrm{C}$ cells showed strong phase advance with increasing contrast, especially to modulation along isoluminant color directions (Fig. $6 A, B$, bottom). We characterized the phase advance by fitting Equations 2-4 to the color direction to which each cell was most responsive and extracting the difference between the response phase a contrast of zero and the maximum contrast attainable (Carandini et al., 1997). To allow comparison of cells characterized with slightly different temporal frequencies we represented the phase difference as a change in latency. In V1, the median latency of group C cells was reduced by $9.7 \mathrm{~ms}$ (SE, $2.5 ; n=14)$, not reliably less than the latency reduction among group A cells ( $11.5 \mathrm{~ms}$; SE, $1.4 ; n=26)$ and group B cells (10.9 ms; SE, $2.8 ; n=19)$.

\section{Comparison of responses and model predictions}

Figures 4-6 show that for many cortical cells the variation of response with contrast along different color directions is consistent with gain regulation from mechanisms with color tuning that differs from that of the LRF. To explore this more fully, we fitted our normalization model to the contrast-response curves. The model requires separate response-scaling terms for each color direction, and our fitting procedure estimated these. Two parameters estimated the vector that determines the contribution of each of the three second-stage mechanisms to the normalizing signal. The remaining parameters are the exponent and the term that determines the overall sensitivity of the pool ( $\sigma$ in Eq. 2$)$. For cells that gave modulated responses (simple cells and most colorpreferring cells), we fitted the model in the complex plane using both response amplitude and phase. For the latter fits we included extra parameters that allowed a phase offset for each contrast-phase curve.

The solid lines in Figure 4 show, for each complex cell, the best-fitting predictions of mean rate obtained from Equation 2. The model performs well, accounting for the shape and height of the individual contrast-response functions. For cells that gave modulated responses, such as those in Figures 5 and 6, and for which we fitted both the amplitude and phase of the response

\footnotetext{
panels show, respectively, response amplitude and phase at the frequency of modulation. $\boldsymbol{B}$ Same as $A$, except for a cell that preferred $L-M$ modulation; the right panels show responses to modulation in the L-M/achromatic plane. C, Same as $\boldsymbol{A}$, for another cell that preferred S-cone modulation. Solid lines are the predictions of the normalization model described in Materials and Methods, fit to response amplitude and phase in the complex plane, for all color directions to which the cell responded (more than are shown). Model and stimulus parameters: for $\boldsymbol{A}, t_{0}=$ $59.3 \mathrm{~ms}, t_{1}=15.4, n=2.3, \theta_{\mathrm{N}}=66.3^{\circ}, \phi_{\mathrm{N}}=72.8^{\circ}, 0.5$ cycles $\cdot$ degree $^{-1}, 5.3 \mathrm{~Hz}$; for $\boldsymbol{B}$, $t_{0}=29.3, t_{1}=9.0, n=4.7, \theta_{\mathrm{N}}=19.1^{\circ}, \phi_{\mathrm{N}}=45.3^{\circ}, 0.0$ cycles $\cdot$ degree $^{-1}, 3.9 \mathrm{~Hz}$; for $C$, $t_{0}=10.1, t_{1}=3.2, n=2.7, \theta_{\mathrm{N}}=43.8^{\circ}, \phi_{\mathrm{N}}=27.1^{\circ}, 0.5$ cycles $\cdot$ degree ${ }^{-1}, 6.0 \mathrm{~Hz}$. Individual parameters estimated the height of each curve. Error bars are \pm 1 SEM from $11(\boldsymbol{A})$ or 20 ( $B, C$ repetitions.
} 
(Eqs. 2-4), the model provided a good account of both response amplitude and phase, although the predictions of response amplitude were no better than those obtained by fitting amplitude alone (data not shown).

The model appears to account well for the responses of simple and complex cells of any chromatic signature, but to be confident of that we need to compare it against simpler variants: a model with a compressive nonlinearity and a linear model. Figure 7 compares the percentage of variance in response left unexplained by the fits to response amplitude only (see Materials and Methods) (Carandini et al., 1997), with corresponding percentage for the linear model (Fig. 7A,B) and the compressive model (Fig. $7 C, D)$. Simple and complex cells were not distinguished by the quality of fits and are not distinguished in the plots. Points above the unit diagonal indicate that the normalization model provided a better overall account. The linear model provided a poor account of the responses of most cells in V1 (Fig. 7A) and V2 (Fig. $7 B)$. The compressive model provided a better account, but the normalization model was still significantly better than the compressive model in explaining fits for all cell groups $(p<0.05 ; t$ tests performed on the logarithm of the ratio). ${ }^{a}$ The normalization model has two more parameters than the compressive model (describing the mechanism weights to the normalization signal) so we should expect it to explain more of the variance. To take this into account, we compared the $\chi^{2}$ norm error returned by each model (see Materials and Methods). For cells in groups B and C, the normalization model was significantly better: the average $\chi_{\text {norm }}^{2}$ errors were, respectively, 0.84 and 0.62 of that for the compressive model $(p<0.05)$. For group $A$, the normalization model was no better than the compressive one.

For $59 \mathrm{~V} 1$ simple cells on which we had reliable measurements of response phase we also fitted the more constrained normalization model (Eqs. 2-4). This was very much better than the compressive model: the unexplained variance was less by a factor of 0.83 for group A cells $(n=26), 0.73$ for group B cells $(n=19)$, and 0.61 for group C cells $(n=14)$. We studied only seven robust simple cells in V2; they too were well fit by the model. We conclude that the normalization model almost always provides a better account of contrast-response curves than do the other models.

\section{Effect of contrast on chromatic signature}

Because the shapes of contrast-response curves vary with the direction of stimulus modulation in color space, the chromatic tuning of a neuron will vary with contrast. To characterize this, we obtained from contrast-response curves the amplitudes of responses to stimuli modulated along different directions in the isoluminant plane at the maximum attainable modulation ("high contrast") and at 0.6 maximum ("mid-contrast"). We included in our analysis only cells that gave responses reliably $>5$ impulses $\cdot \mathrm{s}^{-1}$ to at least one mid-contrast isoluminant stimulus.

\footnotetext{
${ }^{a}$ Across our population of $168 \mathrm{~V} 1$ and $\mathrm{V} 2$ cells, the average error in fitting the normalization model was 0.75 that of the compressive model (for the cells in Figs. 4-6, this was 0.43 ). Yet Figure 7 makes clear that the predictions of the compressive and normalization model were often indistinguishable for group A cells, and the compressive model provided quantitatively better predictions for several cells. Nevertheless, the normalization model provided satisfactory predictions for all cells, whereas the compressive model fails to account for any case in which responses to different color directions saturated at different response levels. The predictions of the two models are similar only when the LRF is aligned with one of the cardinal axes, which is the case for many group A cells. Better predictions provided by the compressive model might reflect a genuine absence of normalization, but it seems more parsimonious to suppose that in such cases the chromatic signature of the normalization pool is not precisely captured by our model. Although the LRF can be assembled from any linear combination of cone inputs, our normalization signal is constrained to arise in the nonlinear combination of signals from three mechanisms that draw with fixed weights on inputs from the different classes of cones. We could have allowed variation in the cone inputs to these mechanisms and achieved better predictions, but this would have made the model less general.
}
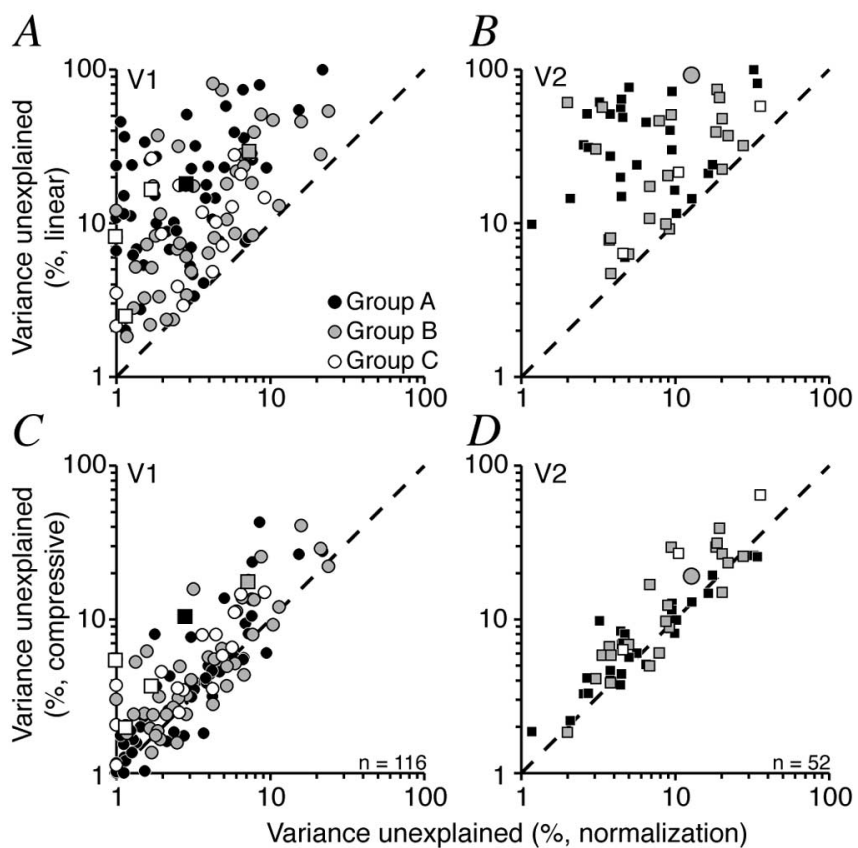

Figure 7. Comparison of the predictive power of three models of response regulation. $\boldsymbol{A}$, Comparison of unexplained variance from fits of the linear and normalization models for V1 neurons in our three groups. The variance left unexplained by each model was obtained from the response amplitude. $\boldsymbol{B}$, Same as $\boldsymbol{A}$, except for V2 neurons. $\boldsymbol{C}$, Comparison of compressive and normalization models for V1 neurons. Other details are the same as for $\boldsymbol{A}$. D, Same as $\boldsymbol{C}$, except for V2 neurons. Points above the unit diagonal indicate that the normalization model provides a better overall description. The squares $(\boldsymbol{A}, \boldsymbol{C})$ and circles $(\boldsymbol{B}, \boldsymbol{D})$ plot the cells shown in Figures $4-6$.

This criterion admitted all but two of 21 group C cells, 21 of 64 group B cells, and 15 of 83 group A cells; the criterion admitted only one neuron that previous work (Johnson et al., 2001, 2004; Solomon et al., 2004) would have characterized as luminance preferring. (The group A cells that responded robustly to chromatic modulation had preferred directions not quite aligned to the achromatic axis and very high modulation sensitivity.)

Figure $8 A-C$ shows the two sets of responses obtained from three V1 cells. For the group C cell in Figure $8 C$, responses increased proportionately with contrast, and the preferred color did not change. For the group $B$ complex cell in Figure $8 B$, response increased disproportionately at azimuth $45^{\circ}$, shifting the preferred azimuth by $14^{\circ}$. For the group A complex cell in Figure $8 \mathrm{~A}$, the two curves have quite different shapes, and the preferred azimuth shifted by $67^{\circ}$. To summarize the data for all cells we calculated the unsigned difference between the preferred azimuths at the two contrast levels. The histograms in Figure $8 D-F$ show this measure for all V1 and V2 cells that met our selection criterion. The median angular difference was $3.9^{\circ}$ for group C cells $(n=19), 9.5^{\circ}$ for group B cells $(n=21)$, and $13.3^{\circ}(n=15)$ for group A cells. These calculations presume reliable measures of the preferred color direction. We used a bootstrap procedure on responses to individual trials to find the $95 \%$ confidence limits on our estimates of the preferred direction. These estimates were less reliable at low contrast (median error, $10.8^{\circ}$ ) than at high contrast $\left(6.1^{\circ}\right)$, but confidence intervals did not overlap for 10 of the 21 cells in which azimuth shifted by $>10^{\circ}$.

These measurements tell us that the preferred azimuth within the isoluminant plane can change dramatically with contrast but say little about how chromatic tuning changes in the full color space of Figure $1 A$, which represents both chromaticity and luminance. Most cortical cells respond better to achromatic mod- 

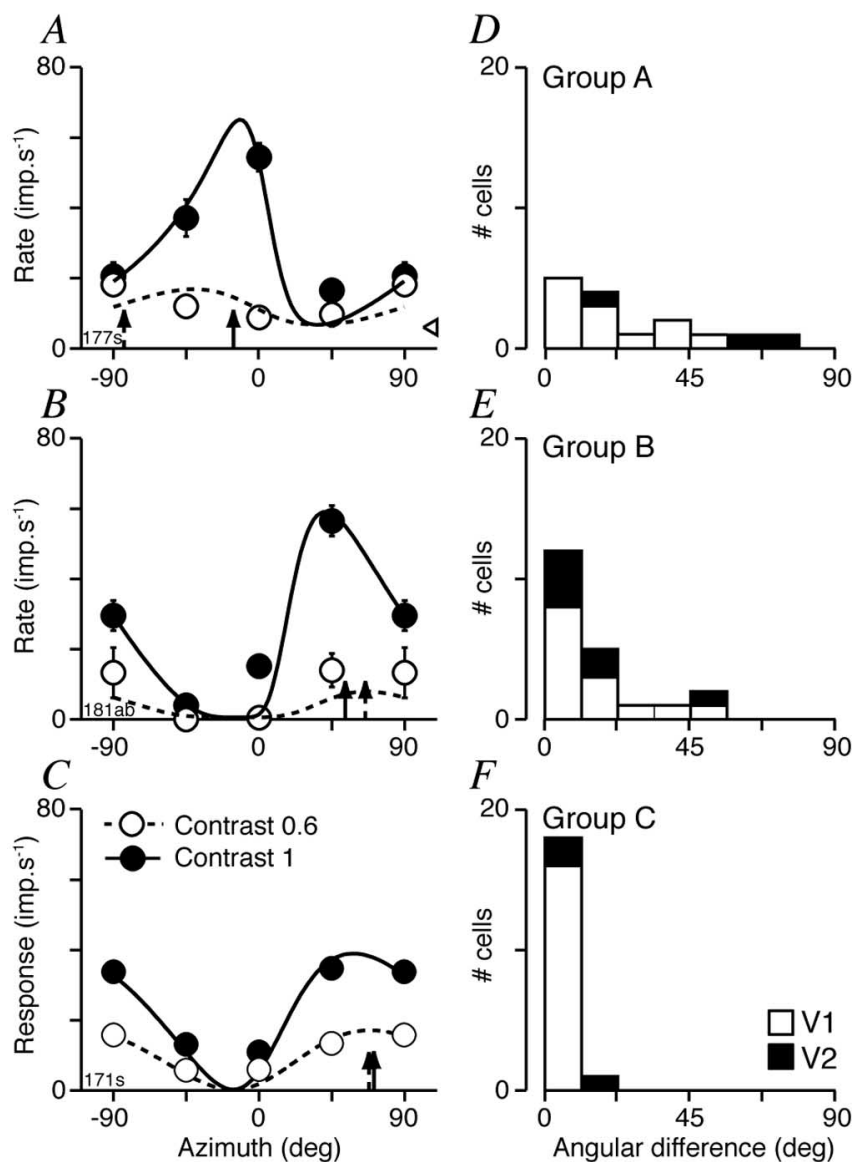

Figure 8. Effect of contrast on chromatic tuning in the isoluminant plane. $\boldsymbol{A}$, Responses of a V2 complex cell (group A) measured at moderate contrast and high contrast. $\boldsymbol{B}, \boldsymbol{C}$, Same as $\boldsymbol{A}$ except for a V1 complex in group B and a V1 color-preferring cell in group C, respectively. Contrast has little effect on the preferred color direction for the group C cell, but for both group $A$ and group $B$ neurons, the change in contrast changed the preferred azimuth (notably so for the cell in $\boldsymbol{A}$ ). Solid lines are the best-fitting predictions of the normalization model described in Results. D, Distributions of unsigned shifts in the preferred azimuth brought about by changing contrast from moderate to high in group A cells in V1 and V2. $E$, Same as $\boldsymbol{D}$, except for group $B$ cells. $\boldsymbol{F}$, Same as $\boldsymbol{D}$, except for group ( cells. Model and stimulus parameters: for $\boldsymbol{A}, \phi_{\mathrm{M}}=$ $-53.6^{\circ}, \phi_{\mathrm{N}}=84.4^{\circ}, \sigma=0.04, n=2.3,2.2$ cycles $^{\circ}$ degree $^{-1}, 5.3 \mathrm{~Hz}$; for $\boldsymbol{B}, \phi_{\mathrm{M}}=76.6^{\circ}$, $\phi_{\mathrm{N}}=90.0^{\circ}, \sigma=0.12, n=5.0,2.9$ cycles $\cdot$ degree $^{-1}, 3.9 \mathrm{~Hz}$; for $C, \phi_{\mathrm{M}}=74.1^{\circ}, \phi_{\mathrm{N}}=$

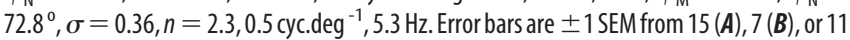
(C) repetitions.

ulation than they do to chromatic modulation, and the contrastresponse functions obtained with achromatic stimuli often saturate at low contrasts. This suggests that contrast might profoundly affect the preferred elevation in the color space. We examined this by fitting the quasilinear model of Equation 9 to modulation sensitivity (obtained as usual from contrast-response curves) along the full range of directions in color space, estimated for the maximum attainable contrast (except along the achromatic axis, where contrast was set at 0.5 ) and one-half of that contrast. The preferred elevation was slightly changed by contrast: median (unsigned) change in elevation was $3.2^{\circ}$ for group C cells $(n=21), 5.0^{\circ}$ for group B cells $(n=64)$, and $5.5^{\circ}$ for group A cells $(n=83)$. For group C cells, elevation shifted toward the isoluminant plane or away from it with equal frequency, and for the group as a whole the average signed shift was only $0.5^{\circ}$ $\left(\mathrm{SD}, 8.3^{\circ}\right)$. For group $\mathrm{A}$ and $\mathrm{B}$ cells, elevation at high contrasts was on average lowered by $2.8^{\circ}\left(\mathrm{SD}, 8.7^{\circ}\right)$ and $3.1^{\circ}\left(\mathrm{SD}, 4.6^{\circ}\right)$, respectively. In some neurons, responses to modulation along direc-
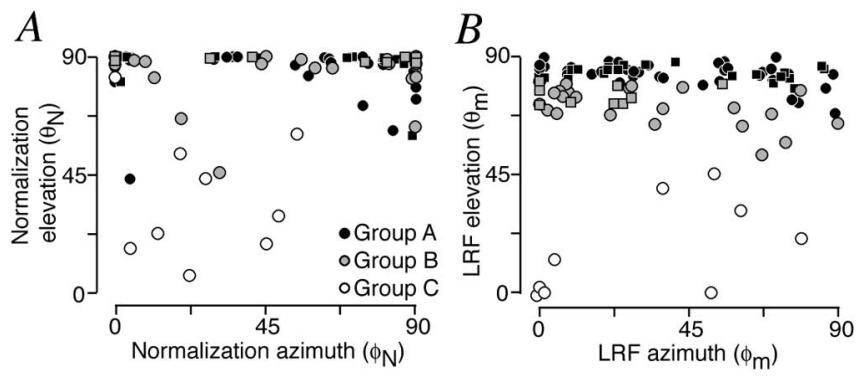

Figure 9. Chromatic signatures of normalization pools and LRFs. $A$, Distribution of preferred directions of normalization pools for cells in which they could be accurately determined ( $n=$ 103). Cell groups $A-C$ are identified by the usual conventions; circles and squares identify, respectively, neurons in V1 and V2. $\boldsymbol{B}$, Same as $\boldsymbol{A}$, except for the LRFs of the same cells.

tions out of the isoluminant plane were already beginning to saturate at the lower of the two contrasts at which we made measurements. To the extent that this happened, our analysis underestimates the change in chromatic preference with contrast.

The contrast-dependent changes in chromatic signature are well captured by the normalization model. The solid lines in Figure $8 A-C$ show the best-fitting predictions of chromatic tuning. For each cell these were obtained by determining the weights with which second-stage mechanisms contribute to the normalization signal and then finding the linear model of cone summation $\left(S_{(\lambda)}\right.$ in Eq. 2; described by Eq. 9$)$ that best described the sets of response amplitudes shown in Figure 8.

\section{Chromatic signature of the normalization pool}

Contrast-dependent changes in chromatic tuning of the kind shown in Figure 8 indicate that the LRF and the normalization pool often have different chromatic signatures. We know a good deal about the signature of the LRF, which can be measured directly (Fig. 2), but we need the model to derive the signature of the normalization pool. For neurons in which response grows almost linearly with contrast this signature is poorly constrained (indeed, such neurons provide no clear evidence of normalization at all), and we need to exclude them from the analysis. We did this by identifying neurons for which the linear model provided a better fit to contrast-response curves. The linear model has two parameters that determine the relationship between chromatic signature and firing rate, whereas the normalization model has five. We therefore included in our analysis only neurons for which the normalization model reduced the unexplained variance to less than two-fifths that left by the linear model, which left $74 \mathrm{~V} 1$ cells ( 9 of which were color-preferring) and $29 \mathrm{~V} 2$ cells.

The preferred elevation $\left(\theta_{\mathrm{N}}\right)$ and azimuth $\left(\phi_{\mathrm{N}}\right)$ of the normalizing signal define its chromatic signature in the same way that equivalent terms define the chromatic signature of the LRF. Figure $9 A$ shows the distribution of these parameters. Because the driving inputs are rectified, vectors that characterize them lie in a single octant: both elevation and azimuth vary between 0 and $90^{\circ}$. For group C cells, the normalizing signal was substantially sensitive to isoluminant modulation [evidently drawing input from both $\mathrm{L}-\mathrm{M}$ and $\mathrm{S}-(\mathrm{L}+\mathrm{M})$ mechanisms] and elevations were lowest. Among other cells the normalizing signal was generally insensitive to isoluminant modulation, and elevations were close to $90^{\circ}$. For comparison, we show in Figure $9 B$ the corresponding data for the LRF (from Fig. 2) folded to the same octant, including only cells that are also represented in Figure 9A. Among cells of groups A and B, the preferred elevation of the normalization pool was generally higher than that of the LRF, notably so for cells 
in group B. This is easily seen in Figure $10 \mathrm{~A}$, which shows, for each cell, the preferred elevation of the normalization pool plotted against the preferred elevation of the LRF. Among the $94 \mathrm{~V} 1$ and V2 neurons in groups $\mathrm{A}$ and $\mathrm{B}$, the median elevation of the normalization pool $\left(89.0^{\circ}\right)$ was significantly higher than that of the LRF $\left(82.3^{\circ}\right)(p<$ 0.001; Wilcoxon rank-sum test).

The preferred azimuths of the normalization pool and LRF often differed substantially. Figure $10 \mathrm{~B}$ shows the distributions of the two preferred azimuths for the 52 neurons in which azimuth could be defined (it is undefined when elevation is $90^{\circ}$ ). To exclude cells in which normalization azimuth was poorly constrained we compared the predictions of the full normalization model to one in which we constrained the elevation of the normalization pool to be $90^{\circ}$. Figure $10 \mathrm{~B}$ includes only neurons for which the full model reduced the unexplained variance to less than twofifths that left by the constrained model (33 V1 cells and 19 V2 cells). For all of these the chromatic signatures of the LRFs lay far enough from the achromatic axis that the preferred azimuths were well defined. Preferred azimuths of LRFs were distributed broadly, among all groups of cells. Preferred azimuths of normalization pools of most group C cells were also distributed broadly, implying robust input from both chromatic mechanisms. In contrast, the preferred azimuths of normalization pools among cells in groups A and $\mathrm{B}$ tended to lie at the upper bounds of the plot, reflecting the fact that (weak) input from chromatic mechanisms was dominated by the one sensitive to S-cone modulation.

The change with contrast in the preferred azimuth of a neuron (Fig. 8) should depend on the chromatic preference of the normalization pool. In $27 \mathrm{~V} 1$ and V2 cells ( 8 in group A, 10 in group B, and 9 in group C) we were able to establish the elevation and azimuth of the normalization pool and also determine the effect of contrast on the preferred azimuth. Among the group $\mathrm{C}$ cells, in which normalization pools generally received strong input from both chromatic mechanisms (median elevation, $29.7^{\circ}$; median distance from nearest cardinal axis, $22.3^{\circ}$ ), the preferred azimuth changed little with contrast (median, $2.3^{\circ}$ ). Among cells of groups A and $\mathrm{B}$, in which the normalization pools were dominated by the achromatic mechanism (median elevation, $88.0^{\circ}$ ) with weak chromatic input dominated by one mechanism (median distance from nearest cardinal axis, $9.4^{\circ}$ ), the preferred azimuth changed substantially $\left(14.6^{\circ}\right)$. Overall, there was a moderate negative correlation between change in the preferred azimuth and

\section{$B$}
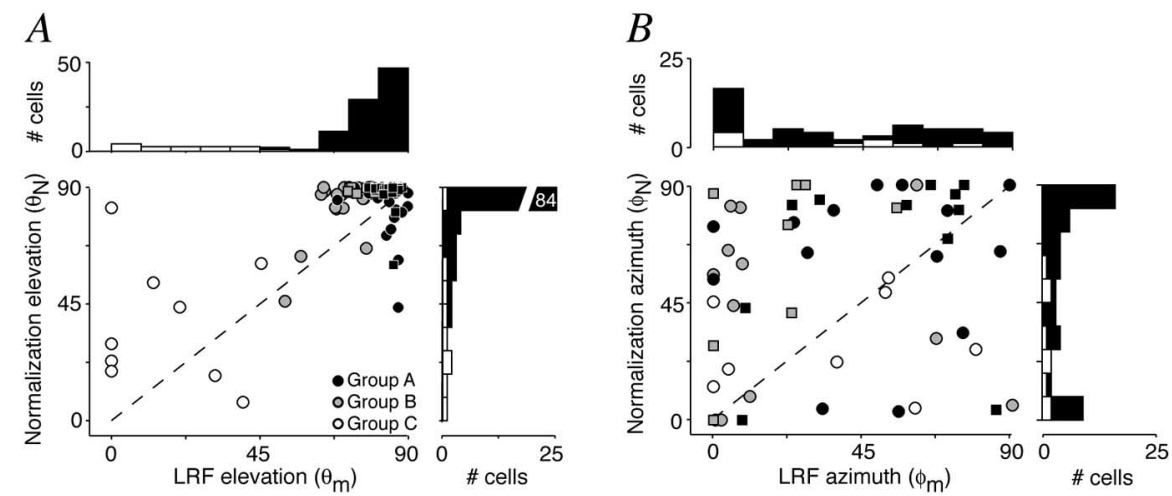

Figure 10. Comparison of chromatic signatures of LRF and normalization pool. $\boldsymbol{A}$, Preferred elevation of normalization pool versus preferred elevation of LRF for cells of Figure 9. Marginal histograms show the distributions of elevations for LRF (top) and the normalization pool (right). The white bars identify group C cells, and the black bars identify group A and B cells. $B$, Preferred azimuth of normalization pool versus preferred azimuth of LRF, for cells in $\boldsymbol{A}$ with preferred elevations of the normalization pool that differed significantly from the achromatic axis $(n=52)$. Marginal histograms show the distributions of azimuths for the LRF (top) and normalization pool (right). Conventions are the same as in $\boldsymbol{A}$.
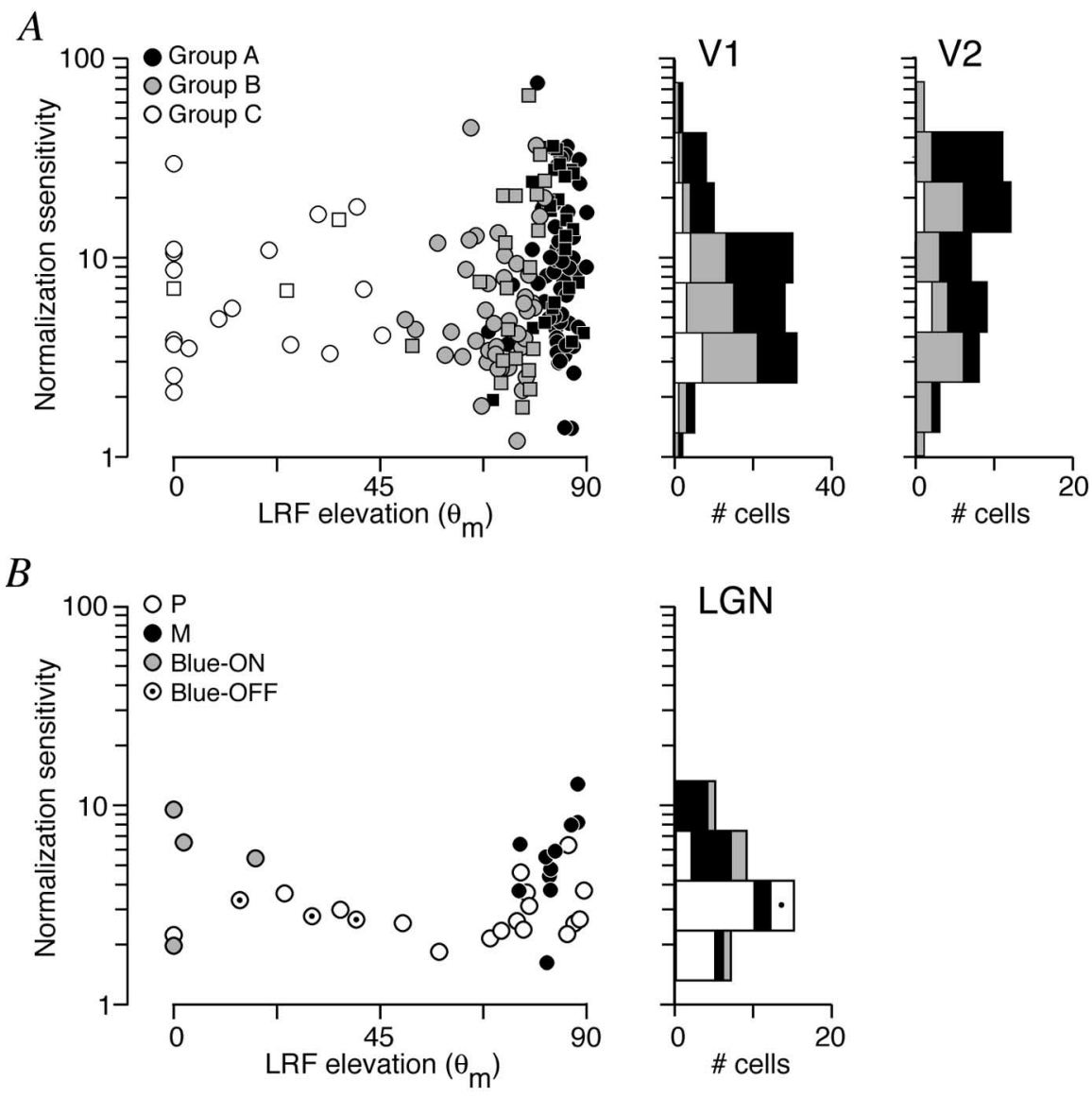

Figure 11. Variation in normalization sensitivity with chromatic signature of the LRF. $A$, Normalization sensitivities of cells in the identified groups A-C in V1 (circles) and V2 (squares) versus the preferred elevation of the LRF. Normalization sensitivity was calculated from fits of the normalization model to the response amplitude. Histograms to the right show the distributions of normalization sensitivities for V1 and V2 cells (black, group A; gray, group B; white, group C). B, Normalization sensitivities for all cells in the LGN versus the preferred elevation of the LRF. The histogram to the right shows the distribution of normalization sensitivity (black, M-cells; gray, blue-ON cells; white, P-cells; white with black dot, blue-OFF cells). the proximity of the normalization pool to the nearest cardinal axis $(r=-0.33 ; p=0.09)$. We conclude that strong, omnidirectional, normalizing signals help stabilize the chromatic tuning of group $\mathrm{C}$ cells and that weaker, more narrowly tuned, normalizing signals destabilize the chromatic tuning of cells in groups A and B. 

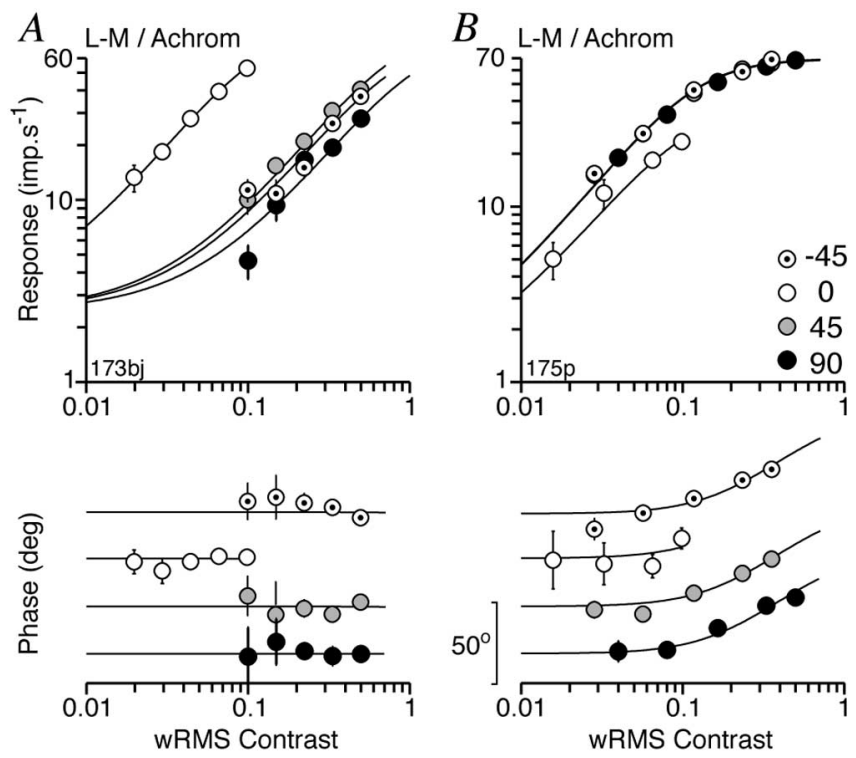

Figure 12. Normalization in P-cells and M-cells in the LGN. Sets of contrast-response curves for a P-cell $(\boldsymbol{A})$ and an M-cell $(\boldsymbol{B})$ are shown. Responses are shown for stimuli modulated along the identified elevations in the $L-M$ /achromatic plane. Conventions are as in Figure 5. Model and stimulus parameters: for $\boldsymbol{A}, t_{0}=0.1 \mathrm{~ms}, t_{1}=11.1, n=1.2, \theta_{\mathrm{N}}=64.1^{\circ}, \phi_{\mathrm{N}}=0.0^{\circ}, 0.0$ cycles $\cdot$ degree $^{-1}, 9.0 \mathrm{~Hz}$; for $\boldsymbol{B}, t_{0}=65.6, t_{1}=7.5, n=1.2, \theta_{\mathrm{N}}=89.4^{\circ}, \phi_{\mathrm{N}}=0.0^{\circ}, 1.0$ cycles $\cdot$ degree $^{-1}, 6.9 \mathrm{~Hz}$. Error bars are $\pm 1 \mathrm{SEM}$ from 11 repetitions.

\section{Strength of normalizing signal and chromatic tuning of the LRF}

Normalization, expressed as saturation of contrast-response curves, is not equally evident in all neurons. In some, particularly group C cells, response amplitude grows almost linearly with contrast. This raises the question of whether the sensitivity of the normalization pool varies with the chromatic signature of the LRF. To estimate sensitivity, we divide the strength of the normalizing signal (the term $\sigma$ in Eq. 2) by the stimulus strength (wRMS contrast; Eq. 7) along the preferred color direction of the normalization pool.

Figure $11 A$ shows the distribution of normalization sensitivity for neurons in V1 and V2, plotted against the preferred elevation of the LRF. On average, normalization sensitivity was higher in V2 neurons (geometric mean, 9.72) than in V1 neurons (6.76; $p<0.02$; Student's $t$ test on the logarithm of the signal strength). In V1, normalization sensitivity was generally higher in group A cells (mean, 7.9; $n=56$ ) than group B (mean, 5.6; $n=42$ ) and group C (mean, $6.3 ; n=18$ ) cells, although not significantly so. Neurons in layer IVB had among the highest normalization sensitivities. Otherwise, there was no obvious relationship between the location and normalization sensitivity of a neuron.

\section{Chromatic properties of normalization in LGN}

Gain-controlling mechanisms akin to normalization have been described in magnocellular (M) cells (Benardete et al., 1992) and, less certainly, in parvocellular (P) cells (Benardete and Kaplan, 1997) in the LGN. We therefore asked whether the normalization observed in cortical neurons could have been inherited from the LGN. We recorded the responses of 42 LGN cells to the stimulus sets we had presented to cortical neurons.

Figure 12 shows, in the format of Figures 5 and 6, the responses of a P-cell (Fig. 12A) to modulation of spatially uniform fields and of an M-cell (Fig. 12B) to drifting gratings of optimal spatial frequency. The solid curves are predictions of the normal-
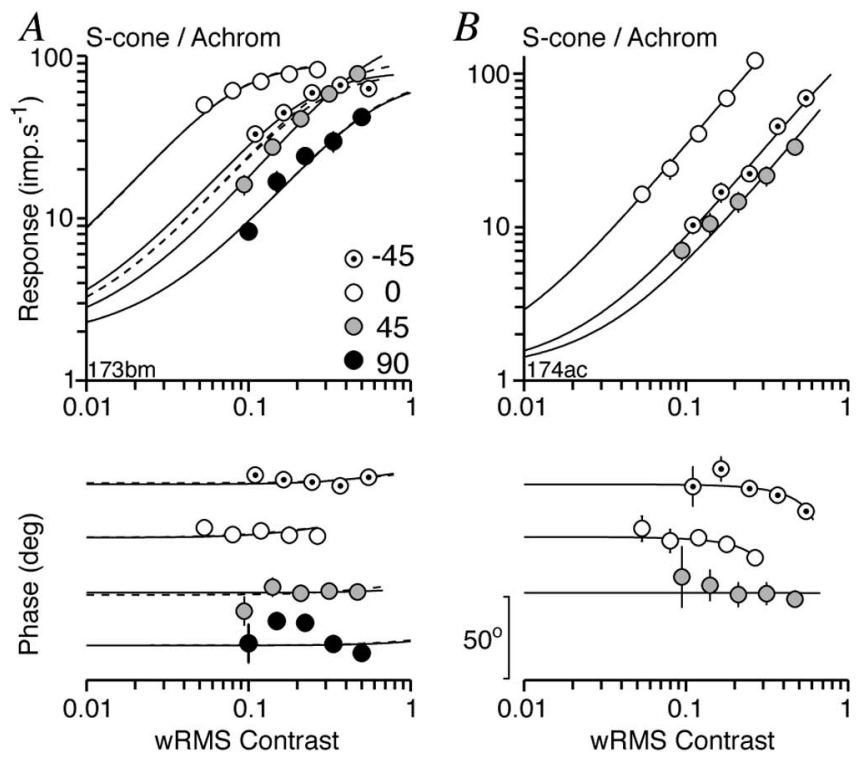

Figure 13. Normalization in S-cone-driven cells in the LGN. Sets of contrast-response curves for a blue-0N cell $(\boldsymbol{A})$ and a blue-OFF cell $(\boldsymbol{B})$. Responses are shown for stimuli modulated along the identified elevations in the $S$-cone/achromatic plane. Conventions are as in Figure 5. Model and stimulus parameters: for $\boldsymbol{A}$ (dashed lines), $t_{0}=6.6 \mathrm{~ms}, t_{1}=3.2, n=1.2, \theta_{\mathrm{N}}=20.8^{\circ}, \phi_{\mathrm{N}}$ $=90.0^{\circ}, 0.0$ cycles $\cdot$ degree ${ }^{-1}, 5.0 \mathrm{~Hz} ;$ for $B, t_{0}=47.1, t_{1}=57.1, n=1.3, \theta_{\mathrm{N}}=0.0^{\circ}, \phi_{\mathrm{N}}=$ $0.0^{\circ}, 0.0$ cycles $\cdot$ degree ${ }^{-1}, 5.0 \mathrm{~Hz}$. Error bars are $\pm 1 \mathrm{SEM}$ from 11 repetitions.

ization model (Eq. 2) used for cortical cells. The P-cell responded robustly to $\mathrm{L}-\mathrm{M}$-modulated fields and less well to achromatic ones. As was the case for some color-preferring cells in V1 and V2, its responses increased almost proportionally with contrast, but without the corresponding phase advance. Among $16 \mathrm{P}$-cells the average phase advance for modulation in the most effective color direction was $1.9 \mathrm{~ms}$ (SE, 1.0), much less than the $9.7 \mathrm{~ms}$ observed for group C cells in V1 ( $p<0.005$; one-sided $t$ test). The M-cell responded best to achromatic gratings and less well (but not negligibly) to isoluminant gratings. As was the case for many group A cells in V1, response amplitude saturated rapidly with increasing achromatic contrast, with a corresponding phase advance. Among $10 \mathrm{M}$-cells, the average phase advance was $7.0 \mathrm{~ms}$ (SE, $1.8)$, slightly less than that for group A cells $(p=0.04)$.

Figure 13 shows the responses of two cells that received strong input from S-cones, one "blue-ON" cell (Fig. 13A) excited by increases in S-cone activation and one "blue-OFF" cell (Fig. 13B) excited by decreases. The differences between the two sets of amplitude-response curves are characteristic of the four blue-ON cells and three blue-OFF cells on which we made full measurements: blue-ON cells were more sensitive and their responses saturated at high contrasts; blue-OFF cells were less sensitive and showed little saturation. Neither type showed clear change in phase with increasing contrast (average phase advance, $1.5 \mathrm{~ms}$; SE, 0.4). The solid curves in Figure $12 \mathrm{~B}$ are the best-fitting predictions of the normalization model (Eq. 2) used for cortical cells. For the blue-ON cell in Figure 13A, and the others on which we had measurements, model fits consistently overestimated the normalization signal along color directions that did not drive $S$ cones well. We therefore considered whether normalization might be driven not by inputs from opponent mechanisms, but by cone signals directly. This simpler model yielded superior fits (Fig. 13A, solid lines) and showed that the weight of the S-cone signal in the normalization pool was, on average, 0.85 , significantly higher than in the LRF (0.55). 

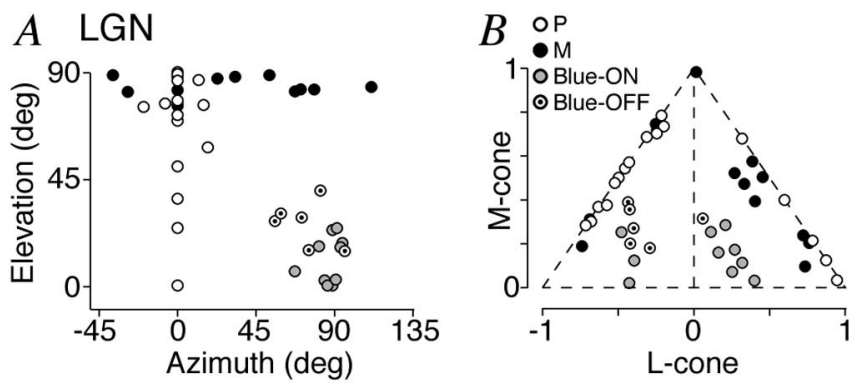

Figure 14. Chromatic signatures of neurons of different classes in the LGN. $A$, Distribution of preferred directions in the color space of Figure $1 A$. B, Relative weights attached to inputs from each cone class. Conventions are as in Figure 2.

Although the normalization model predicted well the contrast-response curves in Figures 12 and 13, fits for P-cells (Fig. $12 A$ ) and blue-OFF cells (Fig. 13B) were poorly constrained because the curves showed little saturation. Moreover, although M-cells showed substantial saturation, and fits were well constrained, predictions were no better than those of the simple compressive model, because the chromatic signature of the putative normalization pool was very like that of the LRF. With these caveats in mind, we can use the fits to estimate normalization sensitivity, as above, for comparison with cortical neurons. Figure $11 B$ shows the distributions of normalization sensitivity for the four types of LGN neurons. The average value was 5.49 for 12 M-cells, 2.91 for 17 P-cells, 5.12 for 4 blue-ON cells, and 2.94 for 3 blue-OFF cells. The values for M-cells are substantially lower than the average values found for cortical cells in groups A and B. The values for P-cells and blue-OFF cells are well below those of almost all cortical neurons. Although the normalization sensitivities of blue-ON cells fell in the range found among group $\mathrm{C}$ neurons, the distinctive chromatic signature of blue-ON cells is not often expressed in the color-preferring cells in V1 (a point we develop below), so normalization in blue-ON cells probably does not contribute to that seen in group C cells. It seems unlikely that normalization in LGN contributes substantially to that observed in the cortex.

Our observations on the LGN provide some new insights into the organization of pathways that convey S-cone signals. Figure $14 \mathrm{~A}$ shows, in the format of Figure 2, the distributions of the preferred azimuths and elevations of all neurons we characterized in the LGN, and Figure $14 \mathrm{~B}$ shows the distribution of weights they assigned to inputs from the different classes of cones. Blue-ON and blue-OFF cells differed in the ways in which they drew on signals from the three classes of cones. Among 10 blue-ON cells, S-cone input (mean weight, $0.55 ; \mathrm{SD}, 0.12$ ) was in seven cases antagonized by a sum of L- and M-cone input; two of the 10 neurons were better fit if the $\mathrm{S}$-cones and $\mathrm{M}$-cones together were opposed by the L-cones, and in the remaining one, S-cones were opposed by L-cones alone. For six blue-OFF cells, the S-cone input (mean weight, $0.38 ; \mathrm{SD}, 0.17$ ) was significantly weaker $(p<0.02)$ and in five of them was in phase with the $\mathrm{M}$-cone input (mean weight, 0.27 ; SD, 0.09). M-cells sometimes responded robustly to $\mathrm{S}$-cone modulation: four of these (each in a different animal) had reasonably strong S-cone input, always in phase with inputs from both $\mathrm{L}$ and $\mathrm{M}$ cones. Other $\mathrm{M}$-cells and $\mathrm{P}$-cells recorded in the same electrode penetrations showed little input from $S$ cones, so it seems unlikely that this behavior resulted from calibration errors.

\section{Discussion}

Chromatic tuning of receptive fields and normalization pools We have shown that among color-preferring cells (group C) the chromatic signature of the normalization pool is omnidirectional in color space and as a result chromatic tuning varies little with contrast. Among all other cells the chromatic signature of the normalization pool lies close to the achromatic axis (Fig. 9A). Because many group A cells prefer achromatic stimuli, their preferences will change little with contrast. Among group A cells with preferences that are not quite aligned to the achromatic axis, and among weakly opponent cells (group B), the chromatic signature of the normalization pool will often differ from that of the LRF, which is relatively more sensitive to chromatic modulation. This causes the chromatic tuning of a neuron to vary with contrast, generally so as to make the neuron relatively more responsive to chromatic modulation as contrast is increased. Even among those group A and B cells with normalization pools that had clear inputs from chromatic mechanisms, the chromatic signature was not stable to variations in contrast. Our analysis suggests that this is because the normalization pool receives signals from only one of the two chromatic mechanisms.

The relative responsiveness of a cell to achromatic and isoluminant stimuli is often taken as an indicator of its potential importance for encoding color (Thorell et al., 1984; Johnson et al., 2001). This ratio will change with contrast if the chromatic signature of the normalization pool is not omnidirectional (a condition clearly met by cells in groups A and B, which will become relatively more responsive to isoluminant stimuli as contrast is increased). The contrast-dependent changes in the signatures of these cells go some way toward explaining apparently conflicting accounts of the proportions of chromatically interesting cells in V1: higher proportions are found in studies that use highcontrast stimuli (Lennie et al., 1990).

Among weakly opponent cells the mismatch between the chromatic signatures of LRFs and normalization pools results principally from variation in the signatures of the LRFs: the signatures of normalization pools are more tightly clustered. We think this variation arises because group B cells in V1 have among the smallest receptive fields (Solomon et al., 2004). A small receptive field that draws inputs from all cones underlying it will be vulnerable to local variations in the arrangement of different types of cones in the mosaic (Roorda et al., 2001): clumping of cones will cause different subregions of the receptive field to have slightly different spectral sensitivities, making the cell weakly color opponent. This local variation will have less influence on the normalization pool, which is presumed to be driven by signals aggregated from all parts of the receptive field.

The instability of chromatic signature in cells that respond relatively well to chromatic modulation (Fig. $9 E, F$ ) is puzzling if their signals are used to convey information about color. However, if their receptive fields are by design the same as those that prefer achromatic modulation, but are simply smaller and therefore more vulnerable to sampling variations, the sensitivity to chromaticity can be viewed as an inevitable but probably inconsequential side effect of their small size. Color-preferring cells (group C), whose high sensitivity to chromatic modulation makes them most obviously well suited to conveying information about color, have the most stable chromatic signatures. Nearly all of these had nonoriented receptive fields with low-pass spatial frequency tuning, although three in V1 and one in V2 had clear spatial selectivity of the kind expected from "double-opponent" receptive fields. 


\section{Origin of normalizing signals}

Two broad classes of biophysical models have been developed to account for normalization: one postulates inhibition arising in the cortex (Heeger, 1992; Carandini et al., 1997; Troyer et al., 1998; Kayser et al., 2001) and the other attributes normalization to synaptic depression of excitatory inputs to the cortex (Abbott et al., 1997; Chance et al., 1998; Carandini et al., 2002).

Our results are incompatible with the most general model of normalization through intracortical inhibition, in which a cell is inhibited by the activity of a large pool of neurons with overlying receptive fields. Were this the case, we would expect the chromatic signature of the normalization pool to be very much the same for every cell, and to resemble the average of the population of LRFs. Although we cannot estimate this average signature precisely (we studied color-preferring neurons preferentially), it is likely to be strongly biased toward the achromatic axis. The normalization pools of color-preferring cells (group C) plainly do not share this signature. The inhibition model might be reconciled with our observations if the normalization pool drew its inputs preferentially from neurons with similar functional characteristics (cf. DeAngelis et al., 1999) or if color-preferring cells occurred in clusters (Livingstone and Hubel, 1987; Ts'o and Gilbert, 1988), thus dominating the normalization pool.

Our results might be more readily accommodated by a model in which normalization arises from synaptic depression of inputs to the cortex. This confers broad tuning for spatial frequency and orientation (Carandini et al., 2002), reflecting the spatial organization of LGN receptive fields. The chromatic properties of group A and B cells suggest little selection of inputs by chromatic signature; the normalization pool will therefore reflect the summed signatures of inputs to both the excitatory and inhibitory regions of the receptive field, which should tend toward the achromatic axis. Inputs to group C cells must be more deliberately organized, so the chromatic signatures of normalization pools will be correspondingly more distinctive.

In LGN receptive fields the preferred elevation shifts toward the achromatic axis as spatial frequency is raised (Derrington et al., 1984), so we asked whether the difference between group C cells and other cells might simply reflect this trend. This did not seem to be the case: our measurements on group C cells were often made with gratings rather than spatially uniform fields, and we saw no sign that the normalization sensitivity varied with the spatial frequency used in the measurement. Moreover, onefourth of our group A and B neurons were characterized with spatial frequencies $<1$ cycles $\cdot$ degree $^{-1}$, and among these groups the preferred elevation of the normalization pool was unrelated to test spatial frequency, even when we considered only cells found in the input layers. Normalization in the cortex cannot simply be inherited from the LGN: normalization in M-cells is not strong enough to account substantially for the behavior of cortical neurons (Fig. $11 A, B$ ), and normalization in blue-ON cells in the LGN has no counterpart among color-preferring cells in the cortex. Nevertheless, normalization in the LGN might contribute to that observed in cortical cells in groups A and B; for example, the strong normalization in blue-ON cells in LGN might contribute to the shift of the preferred azimuth with contrast (Fig. $8 D, E$ ). To explore this, we modeled the effects of linearly summing signals from sets of LGN neurons that had varied response characteristics (e.g., P-cells and M-cells or P-cells and blue-ON cells). This did not capture the behavior of real cortical neurons. A notable feature of the failure was that, unless special control was exercised over the combination of LGN inputs, strongly normalized inputs could give rise to contrast-response functions that were nonmonotonic.

S-cone contributions to receptive fields in the LGN and cortex We were fortunate to record in the LGN from six blue-OFF cells, which are rarely encountered in the retina or LGN (Derrington et al., 1984; Valberg et al., 1986). These cells were appreciably less sensitive than the much more commonly encountered blue-ON cells and showed no normalization. Moreover, they drew differently on inputs from different classes of cones: most blue-OFF cells opposed input from $\mathrm{L}$ cones to inputs from $\mathrm{S}$ and $\mathrm{M}$ cones, whereas blue-ON cells generally opposed inputs from $S$ cones to inputs from $\mathrm{L}$ and $\mathrm{M}$ cones. Our observations are consistent with others from a study in which V1 was silenced to allow recording of thalamic afferents (Chatterjee and Callaway, 2003). Chatterjee and Callaway (2003) found that blue-ON afferents responded robustly to $\mathrm{L}+\mathrm{M}$ modulation, whereas blue-OFF afferents did not, behavior expected if L- and M-cone inputs to blue-OFF cells were of the opposite sign. Our observations are inconsistent with those of Valberg et al. (1986), who found that among blue-OFF cells in the retina, S-cone input was always opposed to $\mathrm{M}$-cone inputs and that L-cone inputs, when present, had the sign of those from S-cones. The disagreement probably reflects differences in the sampling of what might be several morphologically distinct types of blue-OFF cells (Dacey and Packer, 2003).

$\mathrm{S}$-cone inputs to cortical neurons were rarely as strong as those to LGN neurons. Moreover, only 2 of $19 \mathrm{~V} 1$ cells with robust, opponent, $\mathrm{S}$-cone input ( 11 in group $\mathrm{B}$ and 8 in group $\mathrm{C}$ ) showed the chromatic organization $\pm[S-(L+M)]$ found in blue-ON cells in the LGN. The commonest organization found in cortical neurons, $\pm[(S+M)-L]$, was instead that of blue-OFF cells in the LGN, which might be the major source of S-cone input to the cortex. Chatterjee and Callaway (2002) suggested that $S$ cones also contribute, in proportion to their prevalence in the retina, to the receptive fields of $\mathrm{M}$-cells. We found evidence for variable S-cone input to M-cells: S-cone inputs were negligible in seven but substantial in four. Indiscriminate drawing on inputs from all $\mathrm{S}$ cones available in the receptive field might account for this variation among cells.

\section{Relationship to behavioral studies}

Mechanisms underlying the detection and discrimination of patterns defined by modulation along different directions in color space have been explored psychophysically in many studies (for review, see Eskew et al., 1999). Of most relevance here are those that examined the effect of modulation along one direction in color space on the detection and discrimination of modulations along another color-direction (so-called cross-pedestal effects). Chen et al. (2000a,b) and Singer and D'Zmura (1995) showed that at high contrasts modulation along one color direction can increase the contrast-discrimination threshold along any other color direction. Chen et al. (2000a) explained a broad range of psychophysical results with a normalization model much like the one used here: signals pass through linear mechanisms with the preferred directions approximately aligned with the cardinal axes of color space; the sensitivity of each mechanism is regulated by a divisive gain control sensitive to all directions in color space. Such interactions are not found in the LGN but are present in V1. Our observations might also help clarify the spectral tuning of the mechanisms underlying color appearance. The different spectral and contrast sensitivities of cells that receive ON and OFF inputs from $S$ cones suggest that the chromatic signatures of mechanisms revealed by psychophysics will depend on contrast. 


\section{References}

Abbott LF, Varela JA, Sen K, Nelson SB (1997) Synaptic depression and cortical gain control. Science 275:220-224.

Albrecht DG, Hamilton DB (1982) Striate cortex of monkey and cat: contrast response function. J Neurophysiol 48:217-237.

Benardete EA, Kaplan E (1997) The receptive field of the primate P retinal ganglion cell, II: nonlinear dynamics. Vis Neurosci 14:187-205.

Benardete EA, Kaplan E, Knight BW (1992) Contrast gain control in the primate retina: $\mathrm{P}$ cells are not $\mathrm{X}$-like, some $\mathrm{M}$ cells are. Vis Neurosci $8: 483-486$

Bonds AB (1989) Role of inhibition in the specification of orientationselectivity of cells in the cat striate cortex. Vis Neurosci 2:41-55.

Carandini M, Heeger DJ (1994) Summation and division by neurons in primate visual cortex. Science 264:1333-1336.

Carandini M, Heeger DJ, Movshon JA (1997) Linearity and normalization in simple cells of the macaque primary visual cortex. J Neurosci 17:8621-8644.

Carandini M, Heeger DJ, Senn W (2002) A synaptic explanation of suppression in visual cortex. J Neurosci 22:10053-10065.

Chance FS, Nelson SB, Abbott LF (1998) Synaptic depression and the temporal response characteristics of V1 cells. J Neurosci 18:4785-4799.

Chatterjee S, Callaway E (2002) S cone contributions to the magnocellular visual pathway in macaque monkey. Neuron 35:1135.

Chatterjee S, Callaway EM (2003) Parallel colour-opponent pathways to primary visual cortex. Nature 426:668-671.

Chen C, Foley JM, Brainard DH (2000a) Detection of chromoluminance patterns on chromoluminance pedestals I: threshold measurements. Vision Res 40:773-788.

Chen C, Foley JM, Brainard DH (2000b) Detection of chromoluminance patterns on chromoluminance pedestals II: model. Vision Res 40:789-803.

Dacey DM, Packer OS (2003) Colour coding in the primate retina: diverse cell types and cone-specific circuitry. Curr Opin Neurobiol 13:421-427.

DeAngelis GC, Ghose GM, Ohzawa I, Freeman RD (1999) Functional micro-organization of primary visual cortex: receptive field analysis of nearby neurons. J Neurosci 19:4046-4064.

Derrington AM, Krauskopf J, Lennie P (1984) Chromatic mechanisms in lateral geniculate nucleus of macaque. J Physiol (Lond) 357:241-265.

De Valois RL, Cottaris NP, Elfar SD, Mahon LE, Wilson JA (2000) Some transformations of color information from lateral geniculate nucleus to striate cortex. Proc Natl Acad Sci USA 97:4997-5002.

Eskew R, McLellan JS, Giulianini F (1999) Chromatic detection and discrimination. In: Color vision: from genes to perception (Gegenfurtner KR, Sharpe LT, eds), pp 345-368. Cambridge, UK: Cambridge UP.

Geisler WS, Albrecht DG (1992) Cortical neurons: isolation of contrast gain control. Vision Res 32:1409-1410.
Heeger DJ (1992) Normalization of cell responses in cat striate cortex. Vis Neurosci 9:181-197.

Johnson EN, Hawken MJ, Shapley R (2001) The spatial transformation of color in the primary visual cortex of the macaque monkey. Nat Neurosci 4:409-416.

Johnson EN, Hawken MJ, Shapley R (2004) Cone inputs in macaque primary visual cortex. J Neurophysiol 91:2501-2514.

Kayser A, Priebe NJ, Miller KD (2001) Contrast-dependent nonlinearities arise locally in a model of contrast-invariant orientation tuning. J Neurophysiol 85:2130-2149.

Kiper DC, Fenstemaker SB, Gegenfurtner KR (1997) Chromatic properties of neurons in macaque area V2. Vis Neurosci 14:1061-1072.

Krauskopf J, Williams DR, Heeley DW (1982) Cardinal directions of color space. Vision Res 22:1123-1131.

Lennie P, Krauskopf J, Sclar G (1990) Chromatic mechanisms in striate cortex of macaque. J Neurosci 10:649-669.

Levitt JB, Kiper DC, Movshon JA (1994) Receptive fields and functional architecture of macaque V2. J Neurophysiol 71:2517-2542.

Li C, Creutzfeldt O (1984) The representation of contrast and other stimulus parameters by single neurons in area 17 of the cat. Pflügers Arch 401:304-314.

Livingstone MS, Hubel DH (1987) Psychophysical evidence for separate channels for the perception of form, color, movement, and depth. J Neurosci 7:3416-3468.

Roorda A, Metha AB, Lennie P, Williams DR (2001) Packing arrangement of the three cone classes in primate retina. Vision Res 41:1291-1306.

Sclar G, Freeman RD (1982) Orientation selectivity in the cat's striate cortex is invariant with stimulus contrast. Exp Brain Res 46:457-461.

Singer B, D'Zmura M (1995) Contrast gain control: a bilinear model for chromatic selectivity. J Opt Soc Am [A] 12:667-685.

Skottun BC, De Valois RS, Grosof DH, Movshon JA, Albrecht DG, Bonds AB (1991) Classifying simple and complex cells on the basis of response modulation. Vision Res 31:1079-1086.

Solomon SG, Peirce JW, Lennie P (2004) The impact of suppressive surrounds on chromatic properties of cortical neurons. J Neurosci 24:148-160.

Thorell LG, De Valois RL, Albrecht DG (1984) Spatial mapping of monkey V1 cells with pure color and luminance stimuli. Vision Res 24:751-769.

Troyer TW, Krukowski AE, Priebe NJ, Miller KD (1998) Contrast-invariant orientation tuning in cat visual cortex: thalamocortical input tuning and correlation-based intracortical connectivity. J Neurosci 18:5908-5927.

Ts'o DY, Gilbert CD (1988) The organization of chromatic and spatial interactions in the primate striate cortex. J Neurosci 8:1712-1727.

Valberg A, Lee BB, Tigwell DA (1986) Neurones with strong inhibitory $\mathrm{S}$-cone inputs in the macaque lateral geniculate nucleus. Vision Res 26: 1061-1064. 